\title{
Gauge symmetries and holographic anomalies of Chern-Simons and transgression AdS gravity
}

\author{
Pablo Mora \\ Centro Universitario Regional Este (CURE), Universidad de la República, Uruguay, \\ Ruta $9 \mathrm{Km}$ 207, Rocha, Uruguay \\ E-mail: pablomora@cure.edu.uy
}

ABSTRACT: We review the issue of gauge and gravitational anomalies with backgrounds, offering a new outlook on some aspects of these questions.

We compute the holographic anomalies of hypothetical theories dual, in the sense of the AdS-CFT correspondence, to Chern-Simons AdS gravity. Those anomalies are either gauge anomalies, associated to the AdS gauge group of the theory, or diffeomorphism anomalies, with each kind related to the other. AdS gauge anomalies include Weyl, Lorentz (gravitational) and gauge translations while diffeomorphism anomalies include gravitational and Weyl (or scale) anomalies. Our results therefore go beyond previous investigations on Chern-Simons AdS gravity holograpic anomalies, that dealt only with Weyl anomalies. Furthermore, our calculations were done allowing a non vanishing torsion, unlike previous works that considered only the zero torsion, or metric, case.

As a result of using suitable action principles for Chern-Simons AdS gravity, coming from Transgression forms, we obtain finite results without the need for further regularization.

Our results are of potential interest for Lovelock gravity theories, as it has been shown that the boundary terms dictated by the transgressions for Chern-Simons gravity are also suitable to regularize Lovelock theories. The Wess-Zumino consistency condition ensures that anomalies of the generic form computed here should appear for these and other theories.

Keywords: AdS-CFT Correspondence, Chern-Simons Theories, Anomalies in Field and String Theories, Field Theories in Higher Dimensions

ARXIV EPRINT: 1408.1436 


\section{Contents}

1 Introduction $\quad 2$

2 Chern-Simons and Transgression forms and anomalies 4

2.1 Goal of this section 4

2.2 Transgressions 4

2.3 Gauge anomaly with background 5

$\begin{array}{lll}2.4 & \text { Consistent anomaly and covariant anomaly } & 6\end{array}$

$\begin{array}{lll}2.5 & \text { Diffeomorphism anomalies with backgrounds } & 7\end{array}$

2.6 Comparison of the content of this section with previous works on gauge and $\begin{array}{ll}\text { gravitational anomalies } & 8\end{array}$

3 Brief review of transgression and Chern-Simons AdS gravity 9

4 AdS gauge transformations $\quad 11$

4.1 Gauge transformation of the gauge potential 11

$\begin{array}{lll}4.2 & \text { Gauge transformations of the gauge curvature } & 12\end{array}$

$\begin{array}{lll}4.3 & \text { Asymptotic conditions on the gauge parameters } & 13\end{array}$

$\begin{array}{lll}4.3 .1 & \text { Discussion } & 15\end{array}$

5 AdS gauge anomalies of Chern-Simons AdS gravity: backgrounds $\quad \mathbf{1 6}$

$\begin{array}{ll}5.1 \text { AdS gauge anomaly } & 16\end{array}$

$\begin{array}{lll}5.2 & \text { Weyl anomaly } & 17\end{array}$

$\begin{array}{lll}5.3 \text { Lorentz anomaly } & 17\end{array}$

$\begin{array}{ll}5.4 \text { Gauge translations anomaly } & 18\end{array}$

6 AdS gauge anomalies of Chern-Simons AdS gravity: "Kounterterms" 18

$\begin{array}{lll}6.1 & \text { AdS gauge anomaly } & 18\end{array}$

$\begin{array}{ll}6.2 \text { Weyl anomaly } 20 & 20\end{array}$

6.3 Lorentz and translational anomalies 20

7 Diffeomorphism anomalies of Chern-Simons AdS gravity: backgrounds 21

8 Diffeomorphism anomalies of Chern-Simons AdS gravity: "Kounterterms" 21

9 Discussion and comments $\quad 22$ 


\section{Introduction}

Chern-Simons (CS) gravity have been investigated by many researchers during the last few decades, uncovering a wealth of interesting properties in many aspects of these theories (see for instance [1-6]). A recent review with a quite complete list of references is [7].

Within the area of research of Chern-Simons gauge and gravity theories, a number of papers has been devoted to passing from Chern-Simons to transgression forms as a way to address several issues in Chern-Simons theories (see for instance refs. [8-19]). The work discussed here follows the general strategy and outlook advanced in those papers.

The subject of anomalies in quantum field theories is intimately related, for deep mathematical and physical reasons, to the study of Chern-Simons forms [20-23]. We are interested in the possible anomalies of hypothetical theories dual in the sense of the AdSCFT conjecture [24-26] to Chern-Simons AdS gravity.

Holographic anomalies provided some of the first tests of the AdS-CFT correspondence [25, 27], verifying the agreement of the conformal (or Weyl) anomaly [28] for both sides of the duality. This check referred to General Relativity with cosmological constant regulated by suitable boundary terms in 5D on the bulk side and super Yang-Mill theory with $\mathcal{N}=4$ and a large number of colors (related to the cosmological constant of the bulk) in $4 \mathrm{D}$ on the boundary.

Afterwards, conformal anomalies induced by higher curvature gravitational theories in 5D where computed following similar methods [29-31].

The conformal anomaly induced by Chern-Simons gravitational theories was computed in [32] for 5D and 3D CS gravity (inducing Weyl anomalies in 4D and 2D CFTs respectively) and the generic form for arbitrary dimension was conjectured. This calculation was done only on the gravitational side, as the dual CFT theories are not known, but the result of doing this gravitational computation using several different methods was the same, and it was in agreement with what was to be expected from refs. [29-31]. The Weyl anomaly in 4D induced by CS gravity in 5D was also computed in [33] while the Weyl anomaly induced by CS gravity in any dimension was computed in [34]. In those works the Weyl anomaly was computed as the trace of the boundary energy-momentum tensor, adding counterterms to cancel infinite contributions. An analysis of Weyl anomalies for generic gravity theories, including CS gravity, was done in ref. [35], where it was emphasized in particular that the bulk classical field equations are not required to compute the Weyl anomaly. The work of refs. $[36,37]$ deriving the form of Weyl anomalies from the Wess-Zumino consistency conditions and descent equations is also relevant.

In the present work we compute holographic anomalies associated to the AdS gauge invariance of Chern-Simons AdS gravity. We start from the action principles discussed in [38], and assume the asymptotic behavior of the fields assumed in that work. ${ }^{1}$ One important point is that those action principles involve two sets of gauge fields, one of which is regarded as a regulator. The anomaly is then seen as a result of not varying the regulator, as the whole transgression action with both sets of fields varying is in fact gauge

\footnotetext{
${ }^{1}$ This is analogous in principle to the Fefferman-Graham expansion [39], but adapted to a first order formulation allowing a non vanishing bulk torsion.
} 
invariant. A consequence of this built in regulation of the action principles considered is that every magnitude of interest is finite from the beginning, without needing further subtractions or corrections. ${ }^{2}$

Recapitulating, the present work goes beyond the previous works, cited above, on holographic anomalies for Chern-Simons AdS gravity in two relevant ways:

i. While those previous works dealt with the Weyl anomaly only, I study general AdS gauge anomalies, (that include Weyl, Lorentz and gauge translation anomalies), as well as diffeomorphism anomalies (gravitational anomalies and Weyl anomalies). Gravitational or Lorentz anomalies are known to be equivalent [21], and I show that Weyl or scale anomalies are also the same both from the point of view of AdS gauge anomalies and from the point of view of radial diffeomorphisms.

ii. The calculations presented here are done allowing a non vanishing bulk torsion, within the framework developed in [38], while previous calculations were done in the zero torsion, or metric, case. ${ }^{3}$

The plan of this work is the following:

In section 2 I review gauge and diffeomorphism anomalies with backgrounds, giving some new points of view on some aspects of this question.

In section 3 I give a very brief review of Transgression and AdS gravity.

In section $4 \mathrm{I}$ analyze the subset of AdS gauge transformations consistent with the asymptotic fall-off of the fields discussed in [38].

In section 5 AdS holographic gauge anomalies are computed for one of the action principles considered in [38] (the "Backgrounds" action principle).

In section 6 we study AdS holographic gauge anomalies for the second action principle discussed in [38], called "Kounterterms" action principle. ${ }^{4}$

In sections 7 and 8 diffeomorphism anomalies for the "Backgrounds" and "Kounterterms" action principles respectively are discussed.

Section 9 contains a discussion of the results presented and conclusions.

\footnotetext{
${ }^{2}$ Part of the present work may be regarded as an improvement and extension of ref. [40], which dealt with the Weyl anomaly for CS-AdS gravity, following the same general outlook.

${ }^{3}$ Concerning the torsion, in [38] it was found that requiring that the AdS gauge curvature should be asymptotically finite implies that the intrinsic torsion of the boundary must vanish, but that the bulk torsion could be non zero. That the AdS gauge curvature should be finite was found to be a sufficient condition for a well defined action principle and finite conserved quantities. One may interpret those results as suggesting that a boundary theory holograpically related to some bulk theory should have vanishing intrinsic torsion if such a bulk theory has a well posed action principle. That may constitute an explanation of the fact that space-time torsion is negligible in our universe (if the theory that describes it has indeed a holographic formulation). That question, approached from a different perspective (Brane Worlds), is the subject of an interesting recent paper [41].

"The term "Kounterterms" was introduced by R. Olea [42] to distinguish the approach to regularization considered in that paper, and references therein (see also $[18,19,38]$ ), from the standard "Counterterms" approach. We will use the word "Kounterterms" between quotation marks throughout the text, to refer to the approach of [42].
} 


\section{Chern-Simons and Transgression forms and anomalies}

\subsection{Goal of this section}

In this section we review the main results on gauge and gravitational anomalies with backgrounds required in the following sections, with some mathematical preliminaries. Gauge anomalies with backgrounds were studied by Mañes, Stora and Zumino in [22]. What follows could in principle be obtained from that work, nevertheless we find it convenient to derive the explicit forms of the anomalies presented here, and used below. Some of these results were presented in [40]. I am also aware of expressions similar to some of the ones presented here in the work of Moss [43]. Gravitational and Weyl anomalies have been discussed in [21,23] and [28] respectively, but I am not aware of any work presenting diffeomorphism anomalies with backgrounds in the way done in subsection 2.4 below.

The content of this section on gauge and gravitational anomalies applies in principle to any theory involving gauge fields and gravitation, while in the following sections we will apply these results to a specific kind of theories (CS AdS gravity).

\subsection{Transgressions}

Chern-Simons forms $\mathcal{C}_{2 n+1}(A)$ are differential forms defined for a connection $A$, which under gauge transformations transforms by a closed form, and so are said to be quasi invariant. ${ }^{5}$ Transgression forms $\mathcal{T}_{2 n+1}$ are a generalization of Chern-Simons forms that depend on two gauge connections $A$ and $\bar{A}$ and are strictly gauge invariant if both connections are subjected to the same gauge transformation. The use of these forms as Lagrangians for physical theories was discussed in references $[18,19]$. Transgressions can be written (see e.g., [44]) as the difference of two Chern-Simons forms plus an exact form

$$
\mathcal{T}_{2 n+1}(A, \bar{A})=\mathcal{C}_{2 n+1}(A)-\mathcal{C}_{2 n+1}(\bar{A})-d \mathcal{B}_{2 n}(A, \bar{A}),
$$

where $\mathcal{T}_{2 n+1}(A, \bar{A}=0)=\mathcal{C}_{2 n+1}(A)$. The transgression is given explicitly as

$$
\mathcal{T}_{2 n+1}(A, \bar{A})=(n+1) \int_{0}^{1} d t<\Delta A F_{t}^{n}>
$$

In here wedge product between forms are implicit. $A_{t}=t A+(1-t) \bar{A}=\bar{A}+t \Delta A$ is a connection that interpolates between the two independent gauge potentials $A$ and $\bar{A}$. The Lie algebra-valued one-forms $A=A_{A}^{\alpha} G_{\alpha} d x^{A}$ and $\bar{A}=\bar{A}_{A}^{\alpha} G_{\alpha} d x^{A}$ are connections under gauge transformations, $G_{\alpha}$ are the generators of the gauge group $\mathcal{G}$ (a basis of its Lie algebra $\mathfrak{G})$ and $\langle\cdots\rangle$ stands for a symmetric invariant trace in the Lie algebra (or equivalently for the contraction with a symmetric invariant tensor of the group). ${ }^{6}$

\footnotetext{
${ }^{5}$ For the details of the mathematics of Chern-Simons and transgression forms and references see [44].

${ }^{6}$ Notation: upper case Latin indices from the beginning of the alphabet $A, B, C, \ldots$ are space-time indices with values from 0 to $d-1=2 n$; upper case Latin indices from the middle of the alphabet $I, J, K, \ldots$ are space-time indices with values from 0 to $d-1=2 n$ but different from 1 (the index 1 corresponds to a "radial" coordinate, or a coordinate along the direction normal to the boundary); lower case Latin indices from the beginning of the alphabet $a, b, c, \ldots$ are tangent space (or Lorentz) indices with values from 0 to $d-1=2 n$; lower case Latin indices from the middle of the alphabet $i, j, k, \ldots$ are tangent space (or Lorentz) indices with values from 0 to $d-1=2 n$ but different from 1 . The index 1 corresponds to a "radial" direction, or a direction normal to the boundary in tangent space. The index $\alpha$ labels the generators $G_{\alpha}$ of the Lie group considered and takes values from 1 to the dimension of the group.
} 
The corresponding curvature is $F_{t}=d A_{t}+A_{t}^{2}=t F+(1-t) \bar{F}-t(1-t)(\Delta A)^{2}$. Setting $\bar{A}=0$ in the transgression form yields the Chern-Simons form for $A$. If $g$ is an element of $\mathcal{G}$, then a gauge transformation of $A$ is given by $A^{g}=g^{-1}[A+d] g$ and the field strength transforms covariantly as $F^{g}=g^{-1} F g$. If $\bar{A}$ is transformed with the same group element, then $\Delta A$ and $F_{t}$ transform covariantly, and from eq. (2.2) it is clear that the transgression is gauge invariant in that case. The case where $A$ is transformed but $\bar{A}$ is not transformed is considered in the next subsection, and it is relevant to compute gauge anomalies with backgrounds.

\subsection{Gauge anomaly with background}

The variation of the transgression under infinitesimal variations of $A$ and $\bar{A}$ is

$$
\begin{aligned}
\delta \mathcal{T}_{2 n+1}= & (n+1)<F^{n} \delta A>-(n+1)<\bar{F}^{n} \delta \bar{A}> \\
& -n(n+1) d\left\{\int_{0}^{1} d t<\Delta A F_{t}^{n-1} \delta A_{t}>\right\} .
\end{aligned}
$$

We are interested in how the transgression transforms under infinitesimal gauge transformations $g=1+\lambda$ (with $\lambda$ infinitesimal) that change $A$ but not $\bar{A}$. That is, transformations of the form $\delta A=D \lambda$ and $\delta \bar{A}=0$. However, to get the variation of the transgression if only $A$ varies, it is actually easier to exploit the fact that the transgression is invariant if both gauge potentials are varied with the same gauge transformation, and then isolate the part that corresponds to only varying $A$. We start then taking $\delta_{\lambda} A=D \lambda=d \lambda+[A, \lambda]$ and $\delta_{\lambda} \bar{A}=\bar{D} \lambda=d \lambda+[\bar{A}, \lambda]$

$$
\begin{aligned}
0=\delta_{\lambda} \mathcal{T}_{2 n+1}= & +(n+1)<F^{n} D \lambda>-(n+1)<\bar{F}^{n} \bar{D} \lambda>- \\
& -n(n+1) d\left\{\int_{0}^{1} d t<\Delta A F_{t}^{n-1} \delta_{\lambda} A_{t}>\right\} .
\end{aligned}
$$

Using that $\delta_{\lambda} A_{t}=t \delta_{\lambda} A+(1-t) \delta_{\lambda} \bar{A}=t D \lambda+(1-t) \bar{D} \lambda$, the Bianchi identities $D F=0$ and $\overline{D F}=0$, and the property $\langle D$ (something) $\rangle=d<$ something $>$ of the covariant derivative and the invariant trace we get

$$
\begin{aligned}
0=\delta_{\lambda} \mathcal{T}_{2 n+1}= & d\left\{(n+1)<F^{n} \lambda>-n(n+1) \int_{0}^{1} d t t<\Delta A F_{t}^{n-1} D \lambda>\right\}- \\
& -d\left\{(n+1)<\bar{F}^{n} \lambda>-n(n+1) \int_{0}^{1} d t(t-1)<\Delta A F_{t}^{n-1} \bar{D} \lambda>\right\} .
\end{aligned}
$$

But the first line of the last member of the previous equation is just the gauge variation of the transgression if only $A$ is varied, which we will denote $\delta_{\lambda} \mathcal{T}_{2 n+1}^{(A)}$. Therefore

$$
\delta_{\lambda} \mathcal{T}_{2 n+1}^{(A)}=d\left\{(n+1)<\bar{F}^{n} \lambda>-n(n+1) \int_{0}^{1} d t(t-1)<\Delta A F_{t}^{n-1} \bar{D} \lambda>\right\} .
$$

The expression within the brackets on the right hand side of the previous equation is already an expression of the consistent gauge anomaly in the presence of a background $\bar{A}$. By using 
that $\bar{D} \lambda=d \lambda+[\bar{A}, \lambda]$, that $\left\langle\Delta A F_{t}^{n-1} d \lambda>=<d\left[\Delta A F_{t}^{n-1}\right] \lambda>-d<\Delta A F_{t}^{n-1} \lambda>\right.$ and that $d^{2}=0$ we obtain

$$
\begin{aligned}
\delta_{\lambda} \mathcal{T}_{2 n+1}^{(A)}= & d\left\{(n+1)<\bar{F}^{n} \lambda>-n(n+1) \int_{0}^{1} d t(t-1)<d\left[\Delta A F_{t}^{n-1}\right] \lambda>-\right. \\
& \left.-n(n+1) \int_{0}^{1} d t(t-1)<\Delta A F_{t}^{n-1}[\bar{A}, \lambda]>\right\} .
\end{aligned}
$$

Eq. (2.7) differs from eq. (2.6) in that the latter does not include $d \lambda$ while the former does. If we write eq. (2.7) as $\delta_{\lambda} \mathcal{T}_{2 n+1}^{(A)}=d \Omega_{2 n}^{1}(A, \bar{A}, \lambda)$, which defines the anomaly 2 n-form $\Omega_{2 n}^{1}$ in presence of a background, we get

$$
\begin{aligned}
\Omega_{2 n}^{1}(A, \bar{A}, \lambda)= & (n+1)<\bar{F}^{n} \lambda>-n(n+1) \int_{0}^{1} d t(t-1)<d\left[\Delta A F_{t}^{n-1}\right] \lambda>- \\
& -n(n+1) \int_{0}^{1} d t(t-1)<\Delta A F_{t}^{n-1}[\bar{A}, \lambda]>.
\end{aligned}
$$

In the particular case that $\bar{A}=0$ (therefore $\bar{F}=0$ ) eq. (2.8) reduces to

$$
\Omega_{2 n}^{1}(A, \bar{A}=0, \lambda)=-n(n+1) \int_{0}^{1} d t(t-1)<d\left[A F_{t}^{n-1}\right] \lambda>
$$

which agrees with the result $\mathrm{Wu}$, Zee and Zumino for anomalies without backgrounds in ref. [20] (eq. (3.35) in that paper), if we take in account that they define gauge transformations as $\delta_{\lambda} A=-D v$ (so we should replace $\lambda$ by $-v$ ). Another particular case corresponds to choosing $\bar{A}=A$ the $\Delta A=0$ and

$$
\Omega_{2 n}^{1}(A, \bar{A}=A, \lambda)=(n+1)<\bar{F}^{n} \lambda>,
$$

which corresponds to the so called covariant anomaly.

\subsection{Consistent anomaly and covariant anomaly}

If the Quantum Effective Action (also called Quantum Action functional and sometimes denoted $W[A]$ ) of a gauge theory in a space-time of dimension $d$ is denoted $\Gamma[A]$, then its variation under infinitesimal gauge transformations with gauge parameter $\lambda$ defines the Gauge Anomaly d-form $\mathcal{A}[A, \lambda]$

$$
\delta_{\lambda} \Gamma[A]=\int_{\mathcal{M}^{d}} \mathcal{A}[A, \lambda]
$$

where $\mathcal{M}^{d}$ is the space-time manifold [20].

If the anomaly itself were the gauge variation of a local functional $\mathcal{F}[A]$ defined in $\mathcal{M}^{d}$, that is if $\mathcal{A}[A, \lambda]=\delta_{\lambda} \mathcal{F}[A]$, then the anomaly can be absorbed in a redefinition of the quantum action $\Gamma[A] \rightarrow \Gamma[A]-\int_{\mathcal{M}^{d}} \mathcal{F}[A]$, and the anomaly for the new quantum action would be zero.

The definition of the anomaly implies the so called Wess-Zumino consistency condition

$$
\delta_{\eta} \mathcal{A}[A, \lambda]-\delta_{\lambda} \mathcal{A}[A, \eta]=\mathcal{A}[A,[\lambda, \eta]]
$$


where $\lambda$ and $\eta$ are infinitesimal gauge parameters. This condition severely restricts the possible form of the anomaly. The fact that $\delta_{\lambda} \mathcal{T}_{2 n+1}^{(A)}=d \Omega_{2 n}^{1}(A, \bar{A}, \lambda)$ implies that $\Omega_{2 n}^{1}(A, \bar{A}, \lambda)$ satisfies the Wess-Zumino consistency condition, assuming $\bar{A}$ is a fixed background that is not varied under gauge transformations. We have therefore $\mathcal{A}[A, \lambda]=\Omega_{2 n}^{1}(A, \bar{A}, \lambda)$ as a possible consistent anomaly with background $\bar{A}$. That anomaly cannot be absorbed in a redefinition of the quantum action, as it is not the result of a variation of a local functional on $\mathcal{M}^{d}$ but rather is the result of the variation of a functional defined in a $d+1$-dimensional manifold with boundary $\mathcal{M}^{d}$. The previous discussion parallels similar arguments for the case of anomalies without backgrounds (see [20] and references therein).

The covariant anomaly eq. (2.10) does not seem to be consistent (in the sense of the Wess-Zumino consistency condition) at first sight, as it is gauge invariant, and therefore the first member of the consistency condition would vanish while the second member would not. However if we consider eq. (2.8) as the actual definition and $\bar{A}$ as a different field that is set equal to $A$ (for the covariant anomaly) only at the end of the calculation, and that is not to be varied under gauge transformations, then the covariant anomaly is actually consistent.

I believe that any proper definition of the anomaly must include the reference background (even if it were $A=0$ ).

\subsection{Diffeomorphism anomalies with backgrounds}

The non invariance of an action under diffeomorphisms is associated to gravitational and Weyl anomalies. The variation of an arbitrary differential form $\alpha_{p}$ under infinitesimal diffeomorphisms generated by the vector $\xi=\xi^{\mu} \frac{\partial}{\partial x^{\mu}}$, taken at a fixed point, is $\delta_{\xi} \alpha_{p}=$ $\mathcal{L}_{\xi} \alpha_{p}=\left[d I_{\xi}+I_{\xi} d\right] \alpha_{p}$, with $\mathcal{L}_{\xi}$ is the Lie derivative, $d$ is the standard exterior derivative and $I_{\xi}$ is the contraction operator (see for instance [44]). ${ }^{7}$ For a 1-form that is a gauge potential the previous expression is equivalent to $\delta_{\xi} A=D\left[I_{\xi} A\right]+I_{\xi} F .{ }^{8}$

Under infinitesimal diffeomorphisms the Transgression form changes as a differential form should $\delta_{\xi} \mathcal{T}_{2 n+1}=\mathcal{L}_{\xi} \mathcal{T}_{2 n+1}=\left[d I_{\xi}+I_{\xi} d\right] \mathcal{T}_{2 n+1}$ (of course, in a $2 n+1$ dimensional space-time $d \mathcal{T}_{2 n+1}=0$, but we just kept that part as a formal relationship, that would be relevant if said space-time were embedded in a higher dimensional one). The variation of the transgression results from variations from both $A$ and $\bar{A}$, and we could denote it as $\delta_{\xi} \mathcal{T}_{2 n+1}=\delta_{\xi} \mathcal{T}_{2 n+1}^{(A)}+\delta_{\xi} \mathcal{T}_{2 n+1}^{(\bar{A})}$, where the first term of the second member comes from taking the variation of $A$ keeping $\bar{A}$ fixed and the second term to the reverse.

If we understand $\bar{A}$ as a fixed background not to be varied and take variations only of $A$, then the consequent variation of the transgression will have two parts: a normal variation as an ordinary differential form under diffeomorphisms plus an anomalous vari-

${ }^{7}$ The contraction operator $I_{\xi}$ is defined by acting on a p-form $\alpha_{p}$ as

$$
I_{\xi} \alpha_{p}=\frac{1}{(p-1) !} \xi^{\nu} \alpha_{\nu \mu_{1} \ldots \mu_{p-1}} d x^{\mu_{1}} \ldots d x^{\mu_{p-1}}
$$

and being and anti-derivative in the sense that acting on the wedge product of differential forms $\alpha_{p}$ and $\beta_{q}$ of order $p$ and $q$ respectively gives $I_{\xi}\left(\alpha_{p} \beta_{q}\right)=I_{\xi} \alpha_{p} \beta_{q}+(-1)^{p} \alpha_{p} I_{\xi} \beta_{q}$.

${ }^{8}$ And of course $\delta_{\xi} \bar{A}=\bar{D}\left[I_{\xi} \bar{A}\right]+I_{\xi} \bar{F}$. 
ation. Explicitly $\delta_{\xi} \mathcal{T}_{2 n+1}^{(A)}=\delta_{\xi} \mathcal{T}_{2 n+1}-\delta_{\xi} \mathcal{T}_{2 n+1}^{(\bar{A})}$, where $\delta_{\xi} \mathcal{T}_{2 n+1}$ is the normal variation and $-\delta_{\xi} \mathcal{T}_{2 n+1}^{(\bar{A})}$ is the anomalous variation. That variation can be written as

$$
\begin{aligned}
-\delta_{\xi} \mathcal{T}_{2 n+1}^{(\bar{A})}= & I_{\xi}<\bar{F}^{n+1}>+d\left\{(n+1)<\bar{F}^{n} I_{\xi} \bar{A}>-\right. \\
& -n(n+1) \int_{0}^{1} d t(t-1)<\Delta A F_{t}^{n-1} I_{\xi} \bar{F}>- \\
& \left.-n(n+1) \int_{0}^{1} d t(t-1)<\Delta A F_{t}^{n-1} \bar{D} I_{\xi} \bar{A}>\right\},
\end{aligned}
$$

or

$$
-\delta_{\xi} \mathcal{T}_{2 n+1}^{(\bar{A})}=I_{\xi}<\bar{F}^{n+1}>+d \Omega_{2 n}^{1}(A, \bar{A}, \xi)
$$

with

$$
\begin{aligned}
\Omega_{2 n}^{1}(A, \bar{A}, \xi)= & (n+1)<\bar{F}^{n} I_{\xi} \bar{A}>- \\
& -n(n+1) \int_{0}^{1} d t(t-1)<\Delta A F_{t}^{n-1} I_{\xi} \bar{F}>- \\
& -n(n+1) \int_{0}^{1} d t(t-1)<\Delta A F_{t}^{n-1} \bar{D} I_{\xi} \bar{A}>.
\end{aligned}
$$

A judicious choice of the background will kill the bulk term $I_{\xi}<\bar{F}^{n+1}>$, the simplest being $\bar{F}=0$, but we will see below other choices better suited for a well defined action principle for Chern-Simons AdS gravity. If $\left\langle\bar{A}>\right.$ is such that $I_{\xi}\left\langle\bar{F}^{n+1}>=0\right.$, then the diffeomorphism anomaly with background is just $\Omega_{2 n}^{1}(A, \bar{A}, \xi)$.

\subsection{Comparison of the content of this section with previous works on gauge and gravitational anomalies}

The purpose of this subsection is to clarify what aspects of this section are new (to the best of my knowledge), or at least differ in point of view or emphasis from previous works on anomalies.

These are:

i. I present a new explicit expression, eq. (2.8), for gauge anomalies with backgrounds. Related, but not identical, expressions were presented in $[40,43]$. The derivation of eq. (2.8), which uses the fact that the transgression is gauge invariant if both $A$ and $\bar{A}$ are varied to pass from the variation of the transgression when only $A$ is varied (the anomaly), to the variation of the transgression when only the background is varied, is also new.

ii. Regarding differences with the standard references on gravitational anomalies (see for instance $[21,23])$, the expression for the variation of the transgression under diffeomorphisms when only the field $A$ is varied, eq. (2.14), and the the expression for the diffeomorphism (general coordinate transformations) anomaly with backgrounds, 
eq. (2.15), are new. Also the derivation, reading the anomaly from the anomalous variation of the transgression when the reference configuration $\bar{A}$ is not varied, is new. ${ }^{9}$ A new feature revealed in this approach is the occurrence of the bulk term $I_{\xi}<$ $\bar{F}^{n+1}>$ in eq. (2.14), which must vanish if eq. (2.15) is indeed a possible consistent anomaly (as it must be a functional of the gauge field and the field strength at the boundary, which is not the variation of a local functional defined on the boundary). The vanishing of that bulk term, which is trivial in the case usually considered, $\bar{A}=0$, imposes conditions on the possible reference field $\bar{A}$, which we show below to be satisfied for our choices of background. It is worthwhile to remark that the choice $\bar{A}=0$ does not lead to a well defined action principle and finite conserved charges for Chern-Simons AdS gravity [18, 19, 38]. Another new feature is the appearance of the term $(n+1)<\bar{F}^{n} I_{\xi} \bar{A}>$ in the expression for the diffeomorphism anomaly eq. (2.15), which would vanish if $\bar{A}=0$ is assumed.

iii. While the equivalence between consistent and covariant anomalies is well known [21], the observation of section 2.4, that the covariant anomaly is also consistent (satisfying the Wess-Zumino consistency condition), if one remembers that it actually involves both the fields $A$ and $\bar{A}$, with $\bar{A}$ set equal to $A$ at the end, is possibly new (although the point that setting $\bar{A}=A$ yields the covariant anomaly was made by Moss [43]).

iv. A well known result, the Bardeen-Zumino theorem [21], states the equivalence between Lorentz and gravitational anomalies. I mention in this section and show in subsection 5.2 an analogous result relating Weyl anomalies with a subset of AdS gauge anomalies.

I regard points ii. and iv. as those of greatest novelty.

\section{Brief review of transgression and Chern-Simons AdS gravity}

The gauge connection for the AdS group in dimension $d=2 n+1$ is given by $A=\frac{\omega^{a b}}{2} J_{a b}+$ $e^{a} P_{a}$ where $\omega^{a b}$ is the spin connection, $e^{a}$ is the vielbein and $J_{a b}$ and $P_{a}$ are the generators of the AdS group (for Lorentz transformations and translations respectively). ${ }^{10}$ One possible symmetric trace, and the only one I will consider in this paper, is the one which is non zero only for one $P$ generator and $n J$ generators, with values

$$
<J_{a_{1} a_{2}} \ldots J_{a_{2 n-1} a_{2 n}} P_{a_{2 n+1}}>=\kappa \frac{2^{n}}{(n+1)} \epsilon_{a_{1} \ldots a_{2 n+1}}
$$

where $\kappa$ is a constant, which together with the AdS group parameter $l$ ("AdS radius") will characterize the theories. In addition to the basis of the algebra spanned by the generators $P_{a}$ and $J_{a b}$ we will use a basis spanned by the generators $P_{1}, P_{i}, P_{i}+J_{1 i}, P_{i}-J_{1 i}$ and $J_{i j}$,

\footnotetext{
${ }^{9}$ As remarked elsewhere in the text, diffeomorphism anomalies contain both Gravitational and Weyl anomalies.

${ }^{10} \mathrm{~A}$ gauge connection has dimensions of $(\text { length })^{-1}$, so it must be $A=\frac{\omega^{a b}}{2} J_{a b}+\frac{e^{a}}{l} P_{a}$ where $l$ is the 'AdS radius'. I set $l=1$ throughout all the present paper. It is easy to reintroduce $l$ using dimensional analysis, if necessary.
} 
with $i$ an index taking any allowed value but 1 . For these generators the only non zero values of the symmetrized trace are

$$
\begin{aligned}
<J_{i_{1} i_{2}} \ldots J_{i_{2 n-1} i_{2 n}} P_{1}> & =\kappa \frac{2^{n}}{(n+1)} \epsilon_{1 i_{1} \ldots i_{2 n}} \\
<J_{i_{1} i_{2}} \ldots J_{i_{2 n-1} i_{2 n-2}}\left(P_{i_{2 n-1}} \pm J_{1 i_{2 n-1}}\right)\left(P_{i_{2 n}} \mp J_{1 i_{2 n}}\right)> & = \pm \kappa \frac{2^{n+1}}{(n+1)} \epsilon_{1 i_{1} \ldots i_{2 n}} .
\end{aligned}
$$

Notice in particular that

$$
<J_{i_{1} i_{2}} \ldots J_{i_{2 n-1} i_{2 n-2}}\left(P_{i_{2 n-1}} \pm J_{1 i_{2 n-1}}\right)\left(P_{i_{2 n}} \pm J_{1 i_{2 n}}\right)>=0 \text {. }
$$

The transgression for the AdS group is [19]

$$
\mathcal{T}_{2 n+1}=\kappa \int_{0}^{1} d t \epsilon\left(R+t^{2} e^{2}\right)^{n} e-\kappa \int_{0}^{1} d t \epsilon\left(\bar{R}+t^{2} \bar{e}^{2}\right)^{n} \bar{e}+d B_{2 n}
$$

where

$$
B_{2 n}=-\kappa n \int_{0}^{1} d t \int_{0}^{1} d s \epsilon \theta e_{t}\left\{t R+(1-t) \bar{R}-t(1-t) \theta^{2}+s^{2} e_{t}^{2}\right\}^{n-1} .
$$

Here $e^{a}$ and $\bar{e}^{a}$ are the two vielbeins and $\omega^{a b}$ and $\bar{\omega}^{a b}$ the two spin connections, $R=d \omega+\omega^{2}$ and $\bar{R}=d \bar{\omega}+\bar{\omega}^{2}$ are the corresponding curvatures, $\theta=\omega-\bar{\omega}$ and $e_{t}=t e+(1-t) \bar{e} .^{11}$

The action for transgressions for the AdS group is chosen to be [19]

$$
I_{\text {Trans }}=\kappa \int_{\mathcal{M}} \int_{0}^{1} d t \epsilon\left(R+t^{2} e^{2}\right)^{n} e-\kappa \int_{\overline{\mathcal{M}}} \int_{0}^{1} d t \epsilon\left(\bar{R}+t^{2} \bar{e}^{2}\right)^{n} \bar{e}+\int_{\partial \mathcal{M}} B_{2 n},
$$

where $\mathcal{M}$ and $\overline{\mathcal{M}}$ are two manifolds with a common boundary, that is $\partial \mathcal{M} \equiv \partial \overline{\mathcal{M}}$. Notice that, as said in the previous section, this is a generalization from the simpler case where $\mathcal{M} \equiv \overline{\mathcal{M}}$.

We have two natural choices of either regarding both $A$ and $\bar{A}$ as dynamical fields, or regarding one of them (let's say $\bar{A}$ ) as a non dynamical background.

The field equations derived from the action of eq. (3.7) are $\left\langle F^{n} G_{\alpha}>=0\right.$ and $<$ $\bar{F}^{n} G_{\alpha}>=0$, or (see for instance [19])

$$
\begin{array}{ll}
\epsilon\left(R+e^{2}\right)^{n}=0, & \epsilon\left(R+e^{2}\right)^{n-1} T=0 \\
\epsilon\left(\bar{R}+\bar{e}^{2}\right)^{n}=0, & \epsilon\left(\bar{R}+\bar{e}^{2}\right)^{n-1} \bar{T}=0 .
\end{array}
$$

If $\bar{A}$ is taken to be non dynamical only the first line of the previous equations should hold.

What will be done in the next sections can be interpreted in two ways:

i. As the variation of the Transgression-Chern-Simons AdS gravity action (in any of its versions) under those gauge transformations that keep the AdS gauge curvature finite, maintaining $\bar{A}$ fixed. In that sense the result can be regarded as the AdS gauge holographic anomaly for that theory.

\footnotetext{
${ }^{11}$ We use a compact notation where $\epsilon$ stands for the Levi-Civita symbol $\epsilon_{a_{1} \ldots a_{d}}$ and wedge products of differential forms are understood, as was done in refs. [18, 19, 45]. For instance: $\epsilon R e^{d-2} \equiv \epsilon_{a_{1} a_{2} \ldots a_{d}} R^{a_{1} a_{2}} \wedge$ $e^{a_{3}} \wedge \ldots \wedge e^{a_{d-2}},\left(\theta^{2}\right)^{a b}=\theta_{c}^{a} \wedge \theta^{c b}$.
} 
ii. As the construction of the consistent gauge anomaly for the AdS group and the invariant tensor (or symmetric trace) given above, useful in principle (with a suitable coefficient) for other theories.

\section{AdS gauge transformations}

In this section we study the generic asymptotic form of the gauge parameters that would generate gauge transformations consistent with the asymptotic conditions in the gauge fields required in [38]. A similar problem has been considered, for different asymptotic conditions, in [33], and more recently in [46] in $2+1$ dimensions.

\subsection{Gauge transformation of the gauge potential}

Given the AdS gauge parameter $\lambda=\frac{1}{2} \lambda^{a b} J_{a b}+\lambda^{a} P_{a}$, the gauge potential $A=\frac{1}{2} \omega^{a b} J_{a b}+$ $e^{a} P_{a}$, the gauge variation $\delta_{\lambda} A=D \lambda=d A+[A, \lambda]$, and the algebra of generators of the AdS group one gets

$$
\begin{aligned}
\delta_{\lambda} A= & \frac{1}{2}\left[\left(d \lambda^{a b}+\omega^{a}{ }_{c} \lambda^{c b}+\omega^{b}{ }_{c} \lambda^{a c}\right)+\left(e^{a} \lambda^{b}-e^{b} \lambda^{a}\right)\right] J_{a b}+ \\
& +\left[\left(d \lambda^{a}+\omega^{a}{ }_{b} \lambda^{b}\right)-\lambda^{a}{ }_{b} e^{b}\right] P_{a},
\end{aligned}
$$

or

$$
\begin{aligned}
\delta_{\lambda} \omega^{a b} & =\left(d \lambda^{a b}+\omega^{a}{ }_{c} \lambda^{c b}+\omega^{b}{ }_{c} \lambda^{a c}\right)+\left(e^{a} \lambda^{b}-e^{b} \lambda^{a}\right)=D \lambda^{a b}+e^{a} \lambda^{b}-e^{b} \lambda^{a} \\
\delta_{\lambda} e^{a} & =\left(d \lambda^{a}+\omega^{a}{ }_{b} \lambda^{b}\right)-\lambda_{b}^{a} e^{b}=D \lambda^{a}-\lambda^{a}{ }_{b} e^{b},
\end{aligned}
$$

where $D \lambda^{a b} \equiv d \lambda^{a b}+\omega^{a}{ }_{c} \lambda^{c b}+\omega^{b}{ }_{c} \lambda^{a c}$ and $D \lambda^{a} \equiv d \lambda^{a}+\omega^{a}{ }_{b} \lambda^{b}$ are the covariant derivatives associated to the spin connection $\omega^{a b}$.

In ref. [38] it was shown that the gauge potential that results from requiring a finite gauge curvature can be cast in the form

$$
\begin{aligned}
A= & e^{r} \hat{e}_{\infty}^{i}\left(P_{i}-J_{1 i}\right)+ \\
& +\frac{1}{2} \hat{\omega}_{\infty}^{i j} J_{i j}+\frac{1}{2} \hat{\tau}^{i}\left(P_{i}-J_{1 i}\right)+d r P_{1}+ \\
& +\frac{1}{2} e^{-r}\left[\hat{\gamma}^{i j}+d r \hat{\omega}_{r}^{i j}\right] J_{i j}-\frac{1}{2} e^{-r} \hat{\zeta}^{i}\left(P_{i}+J_{1 i}\right),
\end{aligned}
$$

(see ref. [38] for notation). It is important for what follows to note that the coefficient of $P_{1}$ does not need to be $d r$, it is enough that it is finite when $r \rightarrow \infty$ in order to yield a finite gauge curvature $F .^{12}$

\footnotetext{
${ }^{12}$ The asymptotic dependence of $A$ on $r$ must be schematically $A \approx e^{r}\left(P_{i}-J_{1 i}\right)+1 J_{i j}+1\left(P_{i}-J_{1 i}\right)+$ $1 P_{1}+e^{-r} J_{i j}+e^{-r}\left(P_{i}+J_{1 i}\right)$.
} 
We are interested in gauge transformations that preserve the finite character of $F$. A generic gauge transformation acting on $A$ of the form of eq. (4.3) yields

$$
\begin{aligned}
\delta_{\lambda} A= & \frac{1}{2} \delta_{\lambda} \omega^{i j} J_{i j}+\delta_{\lambda} e^{1} P_{1}+ \\
& +\left\{\frac{1}{2} \hat{D}_{\infty} \lambda^{(-) i}-e^{r} \lambda^{(-) i}{ }_{j} \hat{e}_{\infty}^{j}-\frac{1}{2} \lambda^{(-) i}{ }_{j} \hat{\tau}^{j}+\right. \\
& \left.\frac{d r}{2}\left[\partial_{r} \lambda^{(-) i}-\lambda^{(-) i}\right]+\frac{e^{-r}}{2}\left[\hat{\gamma}_{j}^{i}+d r \hat{\omega}_{r j}^{i}\right] \lambda^{(-) j}\right\}\left(P_{i}-J_{1 i}\right) \\
& +\left\{\frac{1}{2} \hat{D}_{\infty} \lambda^{(+) i}+\frac{e^{-r}}{2} \lambda^{(+) i} \hat{\zeta}^{j}+\right. \\
& \left.\frac{d r}{2}\left[\partial_{r} \lambda^{(+) i}+\lambda^{(+) i}\right]+\frac{e^{-r}}{2}\left[\hat{\gamma}_{j}^{i}+d r \hat{\omega}_{r j}^{i}\right] \lambda^{(+) j}\right\}\left(P_{i}+J_{1 i}\right),
\end{aligned}
$$

where $\lambda^{( \pm) i}=\lambda^{i} \pm \lambda^{1 i}$ and $\lambda^{( \pm) i}{ }_{j}=\lambda^{i}{ }_{j} \pm \lambda^{1} \delta^{i}{ }_{j}$, and

$$
\begin{aligned}
\delta_{\lambda} \omega^{i j}= & \hat{D}_{\infty} \lambda^{i j}+d r \partial_{r} \lambda^{i j}+e^{r}\left[\hat{e}_{\infty}^{i} \lambda^{(+) j}-\lambda^{(+) i} \hat{e}_{\infty}^{j}\right]+\frac{1}{2}\left[\hat{\tau}^{i} \lambda^{(+) j}-\lambda^{(+) i} \hat{\tau}^{j}\right]+ \\
& +\frac{e^{-r}}{2}\left[\hat{\zeta}^{i} \lambda^{(-) j}-\lambda^{(-) i} \hat{\zeta}^{j}\right]+e^{-r}\left[\hat{\gamma}_{k}^{i}+d r \hat{\omega}_{r k}^{i}\right] \lambda^{k j}+e^{-r}\left[\hat{\gamma}_{k}^{j}+d r \hat{\omega}_{r k}^{j}\right] \lambda^{i k}+ \\
\delta_{\lambda} e^{1}= & d \lambda^{1}-e^{r} \lambda_{i}^{(+)} \hat{e}_{\infty}^{i}-\frac{1}{2} \lambda_{i}^{(+)} \hat{\tau}^{i}-\frac{e^{-r}}{2} \lambda_{i}^{(-)} \hat{\zeta}^{i} .
\end{aligned}
$$

\subsection{Gauge transformations of the gauge curvature}

The gauge variation of the AdS gauge curvature is $\delta_{\lambda} F=[F, \lambda]$. This implies that the components of $F$ transform as

$$
\begin{aligned}
\delta_{\lambda} F^{a} & =F_{b}^{a} \lambda^{b}-\lambda^{a}{ }_{b} F^{b} \\
\delta_{\lambda} F^{a b} & =-\lambda^{a}{ }_{c} F^{c b}-\lambda^{b}{ }_{c} F^{a c}+F^{a} \lambda^{b}-F^{b} \lambda^{a} .
\end{aligned}
$$

Separating the 1 and the $i \neq 1$ components we get

$$
\begin{aligned}
\delta_{\lambda} F^{i} & =F^{i}{ }_{j} \lambda^{j}+F^{i}{ }_{1} \lambda^{1}-\lambda^{i}{ }_{j} F^{j}-\lambda^{i}{ }_{1} F^{1} \\
\delta_{\lambda} F^{1} & =F^{1}{ }_{i} \lambda^{i}-\lambda^{1}{ }_{i} F^{i} \\
\delta_{\lambda} F^{i j} & =-\lambda^{i}{ }_{k} F^{k j}-\lambda^{j}{ }_{k} F^{i k}-\lambda^{i}{ }_{1} F^{1 j}-\lambda^{j}{ }_{1} F^{i 1}+F^{i} \lambda^{j}-F^{j} \lambda^{i} \\
\delta_{\lambda} F^{1 i} & =-\lambda^{1}{ }_{j} F^{j i}-\lambda^{i}{ }_{j} F^{1 j}+F^{1} \lambda^{i}-F^{i} \lambda^{1},
\end{aligned}
$$

or equivalently

$$
\begin{aligned}
\delta_{\lambda} F^{i}= & \frac{1}{2} F_{j}^{(+) i} \lambda^{(+) j}+\frac{1}{2} F_{j}^{(-) i} \lambda^{(-) j}+F_{1}^{i} \lambda^{1}-\lambda_{j}^{i} F^{j} \\
\delta_{\lambda} F^{1}= & \frac{1}{2} F_{i}^{(+)} \lambda^{(-) i}-\frac{1}{2} F_{i}^{(-)} \lambda^{(+) i} \\
\delta_{\lambda} F^{i j}= & -\lambda^{i}{ }_{k} F^{k j}-\lambda^{j}{ }_{k} F^{i k}- \\
& -\frac{1}{2} \lambda^{(+) i} F^{(-) j}-\frac{1}{2} \lambda^{(-) i} F^{(+) j}+\frac{1}{2} \lambda^{(+) j} F^{(-) i}+\frac{1}{2} \lambda^{(-) j} F^{(+) i} \\
\delta_{\lambda} F^{1 i}= & \frac{1}{2} \lambda^{(-) j} F_{j}^{(+) i}-\frac{1}{2} \lambda^{(+) j} F_{j}^{(-) i}-\lambda^{i}{ }_{j} F^{1 j}-F^{i} \lambda^{1},
\end{aligned}
$$

where $F^{( \pm) i}=F^{i} \pm F^{1 i}$ and $F_{j}^{( \pm) i}=F_{j}^{i} \pm F^{1} \delta^{i}{ }_{j}$. 


\subsection{Asymptotic conditions on the gauge parameters}

We must choose what conditions to impose on the asymptotic dependence of the components of $\lambda$ on $r$. That may seem unnecessary, as $\lambda$ corresponds to an infinitesimal gauge transformation. However we may regard $\lambda$ as infinitesimal at any given large but finite $r$, but with an asymptotic dependence that would render it infinite if $r \rightarrow \infty$ and everything else is kept fixed. Considering that the components of $\lambda$ are of the generic form $\sigma f(x, r)$ where $\sigma$ is some infinitesimal parameter, we must deal with two different limits $\sigma \rightarrow 0$ (although never in fact reaching 0 ) and $r \rightarrow \infty$.

I see at least two possible conditions on the asymptotic behavior of $\lambda$ :

Option I. The first possibility is to require that gauge parameter be such that the infinitesimal gauge transformation preserves the generic asymptotic form of $A$ that yields a finite asymptotic $F$. In that case, from the expressions given above for the gauge variation of $A$ and $F$, we see that we must impose the following conditions:

i. The fields $e^{1}$ and $\omega^{i j}$ are kept asymptotically finite by the allowed gauge transformations implies that the functions $\lambda^{(+) i}(x, r)$ are asymptotically of the form $\lambda^{(+) i}(x, r)=e^{-r} \hat{\lambda}^{(+) i}(x, r)$ with $\hat{\lambda}^{(+) i}(x, r)$ asymptotically finite.

ii. The functions $\lambda^{(-) i}(x, r), \lambda^{i j}(x, r)$ and $\lambda^{1}(x, r)$ (and equivalently $\lambda^{(+) i j}(x, r)$ and $\left.\lambda^{(-) i j}(x, r)\right)$ are asymptotically finite.

Allowing $\lambda^{(-) i}(x, r)=e^{r} \lambda^{(-) i}(x, r)$ with $\lambda^{(-) i}(x, r)$ asymptotically finite would preserve the generic asymptotic behavior of $A$, but would make some components of $F$ asymptotically divergent, therefore we forbid that possibility.

We have then

$$
\begin{aligned}
\delta_{\lambda} A= & \frac{1}{2} \delta_{\lambda} \omega^{i j} J_{i j}+\delta_{\lambda} e^{1} P_{1}+ \\
& +\left\{e^{r}\left[-\lambda^{(-) i} \hat{e}_{\infty}^{j}\right]+\frac{d r}{2}\left[\partial_{r} \lambda^{(-) i}-\lambda^{(-) i}\right]+\frac{1}{2} \hat{D}_{\infty} \lambda^{(-) i}-\frac{1}{2} \lambda^{(-) i}{ }_{j} \hat{\tau}^{j}+\right. \\
& \left.+\frac{e^{-r}}{2}\left[\hat{\gamma}_{j}^{i}+d r \hat{\omega}_{r}^{i}{ }_{j}\right] \lambda^{(-) j}\right\}\left(P_{i}-J_{1 i}\right) \\
& +\left\{\frac{e^{-r}}{2}\left[\hat{D}_{\infty} \hat{\lambda}^{(+) i}+\lambda^{(+) i} \hat{\zeta}^{j}+d r \partial_{r} \hat{\lambda}^{(+) i}\right]+\frac{e^{-2 r}}{2}\left[\hat{\gamma}^{i}{ }_{j}+d r \hat{\omega}_{r}^{i}{ }_{j}\right] \hat{\lambda}^{(+) j}\right\}\left(P_{i}+J_{1 i}\right),
\end{aligned}
$$

and

$$
\begin{aligned}
\delta_{\lambda} \omega^{i j}= & \hat{D}_{\infty} \lambda^{i j}+d r \partial_{r} \lambda^{i j}+\left[\hat{e}_{\infty}^{i} \hat{\lambda}^{(+) j}-\hat{\lambda}^{(+) i} \hat{e}_{\infty}^{j}\right]+\frac{e^{-r}}{2}\left[\hat{\zeta}^{i} \lambda^{(-) j}-\lambda^{(-) i} \hat{\zeta}^{j}\right]+ \\
& +\frac{e^{-r}}{2}\left[\hat{\tau}^{i} \hat{\lambda}^{(+) j}-\hat{\lambda}^{(+) i} \hat{\tau}^{j}\right]+e^{-r}\left[\hat{\gamma}_{k}^{i}+d r \hat{\omega}_{r k}^{i}\right] \lambda^{k j}+e^{-r}\left[\hat{\gamma}_{k}^{j}+d r \hat{\omega}_{r k}^{j}\right] \lambda^{i k} \\
\delta_{\lambda} e^{1}= & d \lambda^{1}-\hat{\lambda}_{i}^{(+)} \hat{e}_{\infty}^{i}-\frac{e^{-r}}{2} \lambda_{i}^{(-)} \hat{\zeta}^{i}-\frac{e^{-r}}{2} \hat{\lambda}_{i}^{(+)} \hat{\tau}^{i} .
\end{aligned}
$$

From the preceding expressions we can read off the variations of the different relevant fields. For instance

$$
\delta_{\lambda} \hat{e}_{\infty}^{i}=-\lambda_{\infty}^{(-) i}{ }_{j} \hat{e}_{\infty}^{j}
$$

where $\lambda_{\infty}^{(-) i}{ }_{j}(x)=\lambda^{(-) i}(x, r \rightarrow \infty)$. 
If $\lambda^{1}$ depends only on the $x$ 's, but not on $r$ asymptotically, or more precisely if $\partial_{r} \lambda^{1} \rightarrow 0$ when $r \rightarrow \infty$, we can make $\delta_{\lambda} e^{1}=0$ asymptotically by choosing the $\hat{\lambda}_{i}^{(+)}(x, r \rightarrow \infty)$ to be the components of $d \lambda^{1}$ in the basis $\hat{e}_{\infty}^{i} \cdot{ }^{13}$ Making $\delta_{\lambda} e^{1}=0$ everywhere would require gauge parameters that are dependent on the specific field configuration. We will not make that sort of choice. If $\delta_{\lambda} e^{1}=0$ at the boundary the gauge potential could in principle be transformed to the form with which we started, with $e^{1}=d r$, by a change of coordinates that reduces to the identity at the boundary.

Option II. The second possibility is to require that the asymptotic behavior of the gauge parameters is such that that the gauge Noether's charge densities are finite, which may be achieved by requiring that it is the same as the asymptotic behavior of $I_{\xi} A$.

From the invariance of the action under diffeomorphisms generated by an infinitesimal space-time vector $\xi^{\mu}$ Noether's theorem yields (see for instance [19]) the conserved current

$$
* j_{\xi}=d Q_{\xi}
$$

where the conserved charge density is

$$
Q_{\xi}=+n(n+1) \int_{0}^{1} d t<\Delta A F_{t}^{n-1} I_{\xi} A_{t}>
$$

Analogously, invariance of the action under gauge transformations generated by the algebra-valued gauge parameter $\lambda$ gives, via Noether's Theorem [19], the conserved current

$$
* j_{\lambda}=d Q_{\lambda},
$$

where the conserved charge density is

$$
Q_{\lambda}=+n(n+1) \int_{0}^{1} d t<\Delta A F_{t}^{n-1} \lambda>
$$

We see that both expressions are the same if we replace $I_{\xi} A_{t}$ by $\lambda$. Therefore, using the results of ref. [38], where it was shown that the asymptotic conditions given ensure a finite $Q_{\xi}$, if the asymptotic behavior of $\lambda$ is the same that of $I_{\xi} A_{t}$ then $Q_{\lambda}$ will be finite. Reasoning as in ref. [38] one can see that the asymptotic behavior of option I gives vanishing $Q_{\lambda}$ 's.

This weaker condition implies that the asymptotic dependence on $A$ is preserved, but also that some components of $F$ would diverge if we take the $r \rightarrow \infty$ limit while keeping $\sigma$ (the infinitesimal factor mentioned above) fixed. In fact, as we are considering infinitesimal transformations, we must regard $\sigma$ as small enough for a given $r$ that the gauge variation of $F$ is in fact infinitesimal (therefore keeping $F$ finite).

From the expressions for $A$ and for the gauge variation of $A$ we see that we must require:

i. The functions $\lambda^{i j}(x, r)$ and $\lambda^{1}(x, r)$ and equivalently $\lambda^{(+) i j}(x, r)$ and $\lambda^{(-) i j}(x, r)$ are asymptotically finite.

\footnotetext{
${ }^{13}$ Whether or not we make this choice will not affect the anomalies computed below.
} 
ii. The functions $\lambda^{(+) i}(x, r)$ are asymptotically of the form $\lambda^{(+) i}(x, r)=e^{-r} \hat{\lambda}^{(+) i}(x, r)$ with $\hat{\lambda}^{(+) i}(x, r)$ asymptotically finite.

iii. The functions $\lambda^{(-) i}(x, r)$ are asymptotically of the form $\lambda^{(-) i}(x, r)=e^{r} \hat{\lambda}^{(-) i}(x, r)$ with $\hat{\lambda}^{(+) i}(x, r)$ asymptotically finite.

We have then

$$
\begin{aligned}
\delta_{\lambda} A= & \frac{1}{2} \delta_{\lambda} \omega^{i j} J_{i j}+\delta_{\lambda} e^{1} P_{1}+ \\
& +\left\{e^{r}\left[\frac{1}{2} \hat{D}_{\infty} \hat{\lambda}^{(-) i}-\lambda^{(-) i} \hat{e}_{\infty}^{j}+\frac{d r}{2} \partial_{r} \hat{\lambda}^{(-) i}\right]-\right. \\
& \left.-\frac{1}{2} \lambda^{(-) i} \hat{\tau}^{j}+\frac{1}{2}\left[\hat{\gamma}^{i}{ }_{j}+d r \hat{\omega}_{r}^{i} j\right] \hat{\lambda}^{(-) j}\right\}\left(P_{i}-J_{1 i}\right) \\
& +\left\{\frac{e^{-r}}{2}\left[\hat{D}_{\infty} \hat{\lambda}^{(+) i}+\lambda^{(+) i} \hat{\zeta}^{j}+d r \partial_{r} \hat{\lambda}^{(+) i}\right]+\frac{e^{-2 r}}{2}\left[\hat{\gamma}_{j}^{i}+d r \hat{\omega}_{r j}^{i}\right] \hat{\lambda}^{(+) j}\right\}\left(P_{i}+J_{1 i}\right),
\end{aligned}
$$

and

$$
\begin{aligned}
\delta_{\lambda} \omega^{i j}= & \hat{D}_{\infty} \lambda^{i j}+d r \partial_{r} \lambda^{i j}+\left[\hat{e}_{\infty}^{i} \hat{\lambda}^{(+) j}-\hat{\lambda}^{(+) i} \hat{e}_{\infty}^{j}\right]+\frac{1}{2}\left[\hat{\zeta}^{i} \hat{\lambda}^{(-) j}-\hat{\lambda}^{(-) i} \hat{\zeta}^{j}\right]+ \\
& +\frac{e^{-r}}{2}\left[\hat{\tau}^{i} \hat{\lambda}^{(+) j}-\hat{\lambda}^{(+) i} \hat{\tau}^{j}\right]+e^{-r}\left[\hat{\gamma}_{k}^{i}+d r \hat{\omega}_{r k}^{i}\right] \lambda^{k j}+e^{-r}\left[\hat{\gamma}_{k}^{j}+d r \hat{\omega}_{r k}^{j}\right] \lambda^{i k} \\
\delta_{\lambda} e^{1}= & d \lambda^{1}-\hat{\lambda}_{i}^{(+)} \hat{e}_{\infty}^{i}-\frac{1}{2} \hat{\lambda}_{i}^{(-)} \hat{\zeta}^{i}-\frac{e^{-r}}{2} \hat{\lambda}_{i}^{(+)} \hat{\tau}^{i} .
\end{aligned}
$$

From the preceding expressions we can read off the variations of the different relevant fields, for instance

$$
\delta_{\lambda} \hat{e}_{\infty}^{i}=\frac{1}{2} \hat{D}_{\infty} \hat{\lambda}^{(-) i}-\lambda_{j}^{(-) i} \hat{e}_{\infty}^{j}+\frac{d r}{2} \partial_{r} \hat{\lambda}^{(-) i}
$$

asymptotically as $r \rightarrow \infty$. If we require that $\delta_{\lambda} \hat{e}_{\infty}^{i}$ has no component along $d r$ we must require that $\partial_{r} \hat{\lambda}^{(-) i} \rightarrow 0$ when $r \rightarrow \infty$.

Making $\delta_{\lambda} e^{1}=0$ at the boundary is less straightforward in this case, as its finite part involves the specific field configuration considered, through $\hat{\zeta}^{i}$, then the only configuration independent choice would be $\hat{\lambda}_{i}^{(-)} \rightarrow 0$ asymptotically, which in fact corresponds to Option I. It seems that in this case we must regard the asymptotic vanishing of $\delta_{\lambda} e^{1}=0$ as a configuration dependent equation that yields configuration dependent asymptotic allowed values for $\hat{\lambda}^{(+) i}$ and $\hat{\lambda}^{(-) i}$

$$
d \lambda^{1}-\hat{\lambda}_{i}^{(+)} \hat{e}_{\infty}^{i}-\frac{1}{2} \hat{\lambda}_{i}^{(-)} \hat{\zeta}^{i}=0
$$

\subsubsection{Discussion}

Option I above is somehow "safer", as $F$ will be kept finite independently of the order in which we take limits, and also allows for a simple configuration independent condition to make $\delta_{\lambda} e^{1}=0$. It has the disadvantage that the Noether charge associated to the gauge invariance vanishes in this case. 
Option II is more general, containing all the gauge transformations allowed by option I and others, and yields finite conserved charges consistent with the Noether charges associated to diffeomorphisms. It has the problems that $\delta_{\lambda} F$ would diverge asymptotically if we are not careful about the order in which limits are taken. Also the condition $\delta_{\lambda} e^{1}=0$ is more involved and configuration dependent in this case.

In the following sections on anomalies we will give the results corresponding to option II. The anomalies corresponding to option I are found by setting $\hat{\lambda}^{(-) i}(x, r \rightarrow \infty)=\hat{\lambda}_{\infty}^{(-) i}=$ 0 in the results for option II.

\section{AdS gauge anomalies of Chern-Simons AdS gravity: backgrounds}

\subsection{AdS gauge anomaly}

We will use the form of the anomaly given in eq. (2.6), in a slightly modified form

$$
\Omega_{2 n}^{1}(A, \bar{A}, \lambda)=(n+1)<\bar{F}^{n} \lambda>-n(n+1) \int_{0}^{1} d t(t-1)<\Delta A F_{t}^{n-1} \delta_{\lambda} \bar{A}>.
$$

For the "AdS vacuum" configuration (see ref. [38]) we have that $\left\langle\bar{F}^{n} \lambda\right\rangle=0$, because it satisfies the classical field equations. We need to see which parts of $\left\langle\Delta A F_{t}^{n-1} \delta_{\lambda} \bar{A}\right\rangle$ do not vanish as a result of the traces or the asymptotic behaviour of the fields. Schematically, the leading order in each generator is

$$
\begin{aligned}
\Delta A & \approx e^{-r} J_{i j}+1\left(P_{i}-J_{1 i}\right)+e^{-r}\left(P_{i}+J_{1 i}\right) \\
F_{t} & \approx 1 J_{i j}+1\left(P_{i}-J_{1 i}\right)+1 P_{1}+e^{-r}\left(P_{i}+J_{1 i}\right) \\
\delta_{\lambda} \bar{A} & \approx e^{r}\left(P_{i}-J_{1 i}\right)+1 J_{i j}+1 P_{1}+e^{-r}\left(P_{i}+J_{1 i}\right) .
\end{aligned}
$$

Proceeding as in ref. [38] we see that there are no divergences and that the only finite contribution comes from taking $\Delta A$ along $\left(P_{i}+J_{1 i}\right)$, all the $F_{t}$ 's along $J_{i j}$ and $\delta_{\lambda} \bar{A}$ along $\left(P_{i}-J_{1 i}\right)$. Using the definition of the symmetric trace we get

$$
\begin{aligned}
\Omega_{2 n}^{1}(A, \bar{A}, \lambda)= & 2 n \kappa \int_{0}^{1} d t(t-1) \epsilon_{i j k_{1} l_{1} \ldots k_{n-1} l_{n-1} \Delta \hat{\zeta}^{i}}\left[\frac{1}{2} \hat{D}_{\infty} \hat{\lambda}^{(-) j}-\lambda_{k}^{(-) j} \hat{e}_{\infty}^{k}\right] \\
& \left(\hat{R}_{\infty}^{k_{1} l_{1}}-\hat{\zeta}_{t}^{k_{1}} \hat{e}_{\infty}^{l_{1}}-\hat{e}_{\infty}^{k_{1}} \hat{\zeta}_{t}^{l_{1}}\right) \ldots\left(\hat{R}_{\infty}^{k_{n-1} l_{n-1}}-\hat{\zeta}_{t}^{k_{n-1}} \hat{e}_{\infty}^{l_{n-1}}-\hat{e}_{\infty}^{k_{n-1}} \hat{\zeta}_{t}^{l_{n-1}}\right) .
\end{aligned}
$$

The AdS vacuum is such that $\hat{R}_{\infty}^{i j}-\hat{\bar{\zeta}}^{i} \hat{e}_{\infty}^{j}-\hat{e}_{\infty}^{i} \hat{\bar{\zeta}}^{j}=0$, then $\hat{R}_{\infty}^{k l}-\hat{\zeta}_{t}^{k} \hat{e}_{\infty}^{l}-\hat{e}_{\infty}^{k} \hat{\zeta}_{t}^{l}=-t\left(\Delta \hat{\zeta}^{k} \hat{e}_{\infty}^{l}+\right.$ $\left.\hat{e}_{\infty}^{k} \Delta \hat{\zeta}^{l}\right)$. Using this in the previous expression we get

$$
\begin{aligned}
& \Omega_{2 n}^{1}(A, \bar{A}, \lambda)=(-1)^{n} 2 n \kappa \int_{0}^{1} d t\left(t^{n}-t^{n-1}\right) \epsilon_{i j k_{1} l_{1} \ldots k_{n-1} l_{n-1}} \Delta \hat{\zeta}^{i}\left[\lambda^{(-) j}{ }_{k} \hat{e}_{\infty}^{k}-\frac{1}{2} \hat{D}_{\infty} \hat{\lambda}^{(-) j}\right] \\
& \left(\Delta \hat{\zeta}^{k_{1}} \hat{e}_{\infty}^{l_{1}}+\hat{e}_{\infty}^{k_{1}} \Delta \hat{\zeta}^{l_{1}}\right) \ldots\left(\Delta \hat{\zeta}^{k_{n-1}} \hat{e}_{\infty}^{l_{n-1}}+\hat{e}_{\infty}^{k_{n-1}} \Delta \hat{\zeta}^{l_{n-1}}\right) .
\end{aligned}
$$

Integrating in the parameter $t$ we get

$$
\begin{aligned}
\Omega_{2 n}^{1}(A, \bar{A}, \lambda)= & \frac{(-1)^{n+1} 2 \kappa}{n+1} \epsilon_{i j k_{1} l_{1} \ldots k_{n-1} l_{n-1}} \Delta \hat{\zeta}^{i}\left[\lambda^{(-) j}{ }_{k} \hat{e}_{\infty}^{k}-\frac{1}{2} \hat{D}_{\infty} \hat{\lambda}^{(-) j}\right] \\
& \left(\Delta \hat{\zeta}^{k_{1}} \hat{e}_{\infty}^{l_{1}}+\hat{e}_{\infty}^{k_{1}} \Delta \hat{\zeta}^{l_{1}}\right) \ldots\left(\Delta \hat{\zeta}^{k_{n-1}} \hat{e}_{\infty}^{l_{n-1}}+\hat{e}_{\infty}^{k_{n-1}} \Delta \hat{\zeta}^{l_{n-1}}\right) .
\end{aligned}
$$


Using $\hat{R}_{\infty}^{i j}-\hat{\bar{\zeta}}^{i} \hat{e}_{\infty}^{j}-\hat{e}_{\infty}^{i} \hat{\bar{\zeta}}^{j}=0$ we can show that $\Delta \hat{\zeta}^{k} \hat{e}_{\infty}^{l}+\hat{e}_{\infty}^{k} \Delta \hat{\zeta}^{l}=-F^{k l}(x, r=\infty) \equiv-F_{\infty}^{k l}$, which implies that the AdS gauge anomaly with backgrounds can be written as

$$
\Omega_{2 n}^{1}(A, \bar{A}, \lambda)=\frac{2 \kappa}{n+1} \epsilon_{i j k_{1} l_{1} \ldots k_{n-1} l_{n-1}} \Delta \hat{\zeta}^{i}\left[\lambda^{(-) j}{ }_{k} \hat{e}_{\infty}^{k}-\frac{1}{2} \hat{D}_{\infty} \hat{\lambda}^{(-) j}\right] F_{\infty}^{k_{1} l_{1}} \ldots F_{\infty}^{k_{n-1} l_{n-1}}
$$

It is important to remark that while we only used the subscript $\infty$ for $F_{\infty}^{k l}$, in fact every function in the previous expression is evaluated at the boundary $r=\infty$.

\subsection{Weyl anomaly}

From eq. (4.13) or eq. (4.21) we see that if the only non zero component of the gauge parameter is $\lambda^{1}$ then

$$
\delta_{\lambda} \hat{e}_{\infty}^{i}=\lambda_{\infty}^{1} \hat{e}_{\infty}^{i}
$$

which implies that this kind of gauge transformation induces a Weyl transformation at the boundary. From eq. (5.6) we see that the Weyl anomaly reads

$$
\begin{aligned}
\Omega_{2 n}^{1}(A, \bar{A}, \lambda) & =-\frac{2 \kappa \lambda_{\infty}^{1}}{n+1} \epsilon_{i j k_{1} l_{1} \ldots k_{n-1} l_{n-1}} \Delta \hat{\zeta}_{\infty}^{i} \hat{e}_{\infty}^{j} F_{\infty}^{k_{1} l_{1}} \ldots F_{\infty}^{k_{n-1} l_{n-1}}= \\
& =-\frac{\kappa \lambda_{\infty}^{1}}{n+1} \epsilon_{i j k_{1} l_{1} \ldots k_{n-1} l_{n-1}}\left[\Delta \hat{\zeta}_{\infty}^{i} \hat{e}_{\infty}^{j}+\hat{e}_{\infty}^{i} \Delta \hat{\zeta}_{\infty}^{j}\right] F_{\infty}^{k_{1} l_{1}} \ldots F_{\infty}^{k_{n-1} l_{n-1}}= \\
& =\frac{\kappa \lambda_{\infty}^{1}}{n+1} \epsilon_{i j k_{1} l_{1} \ldots k_{n-1} l_{n-1}} F_{\infty}^{i j} F_{\infty}^{k_{1} l_{1}} \ldots F_{\infty}^{k_{n-1} l_{n-1}} .
\end{aligned}
$$

The last line would vanish if the field equations hold, but that does not mean that the Weyl anomaly vanishes, because gauge symmetries must hold whether or not the field equations hold (they hold off-shell, not just on-shell). ${ }^{14}$

\subsection{Lorentz anomaly}

From eq. (4.13) or eq. (4.21) we see that if the only non zero component of the gauge parameter is $\lambda^{i j}$ then

$$
\delta_{\lambda} \hat{e}_{\infty}^{i}=-\lambda_{\infty j}^{i} \hat{e}_{\infty}^{j}
$$

which implies that this kind of gauge transformation induces a Lorentz transformation at the boundary. The corresponding anomaly can be interpreted as a Lorentz anomaly (which is equivalent to a gravitational anomaly, as shown in ref. [21]). From eq. (5.6) we see that the Lorentz anomaly reads

$$
\Omega_{2 n}^{1}(A, \bar{A}, \lambda)=\frac{2 \kappa}{n+1} \epsilon_{i j k_{1} l_{1} \ldots k_{n-1} l_{n-1}} \Delta \hat{\zeta}^{i}\left[\lambda_{\infty k}^{j} \hat{e}_{\infty}^{k}\right] F_{\infty}^{k_{1} l_{1}} \ldots F_{\infty}^{k_{n-1} l_{n-1}}
$$

This expression is not necessarily zero even if the field equations hold. It is unclear to me which kind of gravitational coupling in a boundary theory may generate such Lorentzgravitational anomaly (if any). Known gravitational anomalies, that arise in known theories

\footnotetext{
${ }^{14}$ One may however argue that if there exist a holographic theory induced at the boundary which could be approximated by a saddle point approximation of the bulk theory, then the Weyl anomaly for that conjectured holographic boundary theory in that regime would vanish.
} 
with chiral fermions or anti symmetric self dual (or anti self dual) tensors, as discussed in refs. [21, 23] (see also references in [23]), are associated to symmetric standard traces or products of standard traces, rather than to the symmetric trace associated to the LeviCivita tensor. ${ }^{15}$ That is also true for gravitational anomalies considered in works in the holographic context, for instance refs. [48, 49].

It would be interesting to know if there exists a kind of theories that would have a gravitational anomaly of the form given in eq. (5.10), or to know a reason why such theories do not exist.

\subsection{Gauge translations anomaly}

If the only non zero components of the gauge parameter are $\hat{\lambda}^{(-) i}$ we could in principle have a gauge translation anomaly, which using eq. (5.6) would be

$$
\Omega_{2 n}^{1}(A, \bar{A}, \lambda)=-\frac{\kappa}{n+1} \epsilon_{i j k_{1} l_{1} \ldots k_{n-1} l_{n-1}} \Delta \hat{\zeta}^{i}\left[\hat{D}_{\infty} \hat{\lambda}^{(-) j}\right] F_{\infty}^{k_{1} l_{1}} \ldots F_{\infty}^{k_{n-1} l_{n-1}} .
$$

Notice that for the Option I of the asymptotic behavior of the gauge parameter the previous expression vanishes, and therefore in that case there are no anomalies associated to gauge translations in the backgrounds approach to Chern-simons AdS gravity.

\section{AdS gauge anomalies of Chern-Simons AdS gravity: "Kounterterms"}

\subsection{AdS gauge anomaly}

We will again use the form of the anomaly given by eq. (5.1).

For the "Kounterterms vacuum" configuration we have $\bar{A}=\frac{1}{2} \hat{\omega}_{\infty}^{i j} J_{i j}+d r P_{1}$ and $\bar{F}=$ $\frac{1}{2} \hat{R}_{\infty}^{i j} J_{i j}$ (see ref. [38]), therefore $<\bar{F}^{n} \lambda>$ has a non-zero contribution from the component of $\lambda$ along $P_{1}$, which is

$$
(n+1)<\bar{F}^{n} \lambda>=\kappa \epsilon_{k_{1} l_{1} \ldots k_{n} l_{n}} \hat{R}_{\infty}^{k_{1} l_{1}} \ldots \hat{R}_{\infty}^{k_{n} l_{n}} \lambda^{1}=\kappa E_{n} \lambda^{1}
$$

where $E_{n}=\epsilon_{k_{1} l_{1} \ldots k_{n} l_{n}} \hat{R}_{\infty}^{k_{1} l_{1}} \ldots \hat{R}_{\infty}^{k_{n} l_{n}}$ is the Euler density of the boundary.

In order to compute the non zero contributions to the anomaly coming from $<$ $\Delta A F_{t}^{n-1} \delta_{\lambda} \bar{A}>$ we need the asymptotic behaviour of the relevant fields, which schematically is

$$
\begin{aligned}
\Delta A & \approx e^{-r} J_{i j}+e^{r}\left(P_{i}-J_{1 i}\right)+e^{-r}\left(P_{i}+J_{1 i}\right) \\
F_{t} & \approx 1 J_{i j}+1\left(P_{i}-J_{1 i}\right)+1 P_{1}+e^{-r}\left(P_{i}+J_{1 i}\right) \\
\delta_{\lambda} \bar{A} & \approx 1 J_{i j}+e^{r}\left(P_{i}-J_{1 i}\right)+1 P_{1}+e^{-r}\left(P_{i}+J_{1 i}\right) .
\end{aligned}
$$

\footnotetext{
${ }^{15}$ The basic invariant tensors of $\mathrm{SO}(D)$ groups (of any signature) are the Levi-Civita tensor with $D$ indices and the Minkowski $\eta$ tensor (of the right signature). The symmetric traces in the algebra of the group, denoted in the text by $\langle\ldots\rangle$, are either the Levi-Civita tensor, or a combination of Minkowski $\eta$ with the right symmetry (which can also be obtaining as a combination of standard traces of the generators). See for instance ref. [7] and references therein about this point. The anomalies are characterized by the group, the symmetric trace $\langle\ldots\rangle$, and a global constant factor.
} 
This asymptotic behavior results from from the expressions for $\Delta A$ and $F_{t}$ given in ref. [38] and from

$$
\begin{aligned}
\delta_{\lambda} \bar{A}= & \frac{1}{2}\left[\hat{D}_{\infty} \lambda^{i j}+d r \partial_{r} \lambda^{i j}\right] J_{i j}+\frac{e^{r}}{2}\left[\hat{D}_{\infty} \hat{\lambda}^{(-) i}+d r\left(\partial_{r} \hat{\lambda}^{(-) i}+\hat{\lambda}^{(-) i}\right)\right]\left(P_{i}-J_{1 i}\right)+ \\
& +\frac{e^{-r}}{2}\left[\hat{D}_{\infty} \lambda^{(+) i}+d r\left(\partial_{r} \hat{\lambda}^{(+) i}-\hat{\lambda}^{(+) i}\right)\right]\left(P_{i}+J_{1 i}\right)+\left[\hat{d} \lambda^{1}+d r \partial_{r} \lambda^{1}\right] P_{1}
\end{aligned}
$$

Proceeding as in the previous section we see that there are no divergent contributions to $<\Delta A F_{t}^{n-1} \delta_{\lambda} \bar{A}>$ that come from taking:

i. $\Delta A$ along $\left(P_{i}-J_{1 i}\right)$, all the $F_{t}$ 's along $J_{i j}$ and $\delta_{\lambda} \bar{A}$ along $\left(P_{i}+J_{1 i}\right)$ or

ii. $\Delta A$ along $\left(P_{i}-J_{1 i}\right)$, one of the $F_{t}$ 's along $\left(P_{i}+J_{1 i}\right)$, the remaining $F_{t}$ 's but one along $J_{i j}$ and $\delta_{\lambda} \bar{A}$ along $J_{i j}$ or

iii. $\Delta A$ along $\left(P_{i}+J_{1 i}\right)$, all the $F_{t}$ 's along $J_{i j}$ and $\delta_{\lambda} \bar{A}$ along $\left(P_{i}-J_{1 i}\right)$.

Using the explicit forms of the relevant fields and the definition of the symmetric trace we get

$$
\begin{aligned}
& \Omega_{2 n}^{1}(A, \bar{A}, \lambda)=\kappa \epsilon_{k_{1} l_{1} \ldots k_{n} l_{n}} \hat{R}_{\infty}^{k_{1} l_{1}} \ldots \hat{R}_{\infty}^{k_{n} l_{n}} \lambda^{1}+ \\
& +2 n \kappa \int_{0}^{1} d t(t-1) \epsilon_{i j k_{1} l_{1} \ldots k_{n-1} l_{n-1}} \hat{e}_{\infty}^{i}\left[\hat{D}_{\infty} \hat{\lambda}^{(+) j}\right] F_{t}^{k_{1} l_{1}} \ldots F_{t}^{k_{n-1} l_{n-1}}+ \\
& +2 n \kappa \int_{0}^{1} d t(t-1) t \epsilon_{i j k_{1} l_{1} \ldots k_{n-1} l_{n-1}} \hat{e}_{\infty}^{i}\left[\hat{D}_{\infty} \hat{\zeta}^{j}\right] F_{t}^{k_{1} l_{1}} \ldots F_{t}^{k_{n-2} l_{n-2}}\left[\hat{D}_{\infty} \lambda^{k_{n-1} l_{n-1}}\right]- \\
& -n \kappa \int_{0}^{1} d t(t-1) \epsilon_{i j k_{1} l_{1} \ldots k_{n-1} l_{n-1}} \hat{\zeta}_{\infty}^{i}\left[\hat{D}_{\infty} \hat{\lambda}^{(-) j}\right] F_{t}^{k_{1} l_{1}} \ldots F_{t}^{k_{n-1} l_{n-1}}
\end{aligned}
$$

where we ignored contributions along $d r$, as they have no support at the boundary, we used that $F_{t}^{k l}=\hat{R}_{\infty}^{k l}-t^{2}\left(\hat{\zeta}^{k} \hat{e}_{\infty}^{l}+\hat{e}_{\infty}^{k} \hat{\zeta}^{l}\right)$, and every field that appears is evaluated at $r \rightarrow \infty$.

It is possible, integrating by parts, to write the expression for the anomaly in such a way that none of the gauge parameters are acted upon by derivatives. As it was said above, given a symmetric trace with all indices saturated $\langle\ldots\rangle$ and a covariant derivative $D$ it holds that $d<$ (something) $>=<D$ (something) $>$, where $d$ stands for the exterior derivative. This is true in particular for de symmetric trace provided by contraction with the Levi-Civita $\epsilon$-tensor and the covariant derivative $\hat{D}_{\infty}$. Furthermore $\hat{D}_{\infty} \hat{e}_{\infty}^{i}=0$ because of the required vanishing of the intrinsic torsion of the boundary, and $\hat{D}_{\infty} \hat{R}_{\infty}^{i j}=0$ in virtue of the Bianchi identities. We have

$$
\begin{aligned}
\hat{D}_{\infty} F_{t}^{k l} & =-t^{2}\left[\left(\hat{D}_{\infty} \hat{\zeta}^{k}\right) \hat{e}_{\infty}^{l}-\hat{e}_{\infty}^{k}\left(\hat{D}_{\infty} \hat{\zeta}^{l}\right)\right] \\
\hat{D}_{\infty}\left(\hat{D}_{\infty} \hat{\zeta}^{i}\right) & =\hat{R}_{\infty j}^{i} \hat{\zeta}^{j}
\end{aligned}
$$

and

$$
\begin{aligned}
& d\left[\epsilon_{i j k_{1} l_{1} \ldots k_{n-1} l_{n-1}} \hat{e}_{\infty}^{i} \hat{\lambda}^{(+) j} F_{t}^{k_{1} l_{1}} \ldots F_{t}^{k_{n-1} l_{n-1}}\right]= \\
& =-\epsilon_{i j k_{1} l_{1} \ldots k_{n-1} l_{n-1}} \hat{e}_{\infty}^{i}\left[\hat{D}_{\infty} \hat{\lambda}^{(+) j}\right] F_{t}^{k_{1} l_{1}} \ldots F_{t}^{k_{n-1} l_{n-1}}+ \\
& \quad-(n-1) \epsilon_{i j k_{1} l_{1} \ldots k_{n-1} l_{n-1}} \hat{e}_{\infty}^{i} \hat{\lambda}^{(+) j}\left[\hat{D}_{\infty} F_{t}^{k_{1} l_{1}}\right] F_{t}^{k_{2} l_{2}} \ldots F_{t}^{k_{n-1} l_{n-1}}
\end{aligned}
$$


and also

$$
\begin{aligned}
& d\left\{\epsilon_{i j k_{1} l_{1} \ldots k_{n-1} l_{n-1}} \hat{e}_{\infty}^{i}\left[\hat{D}_{\infty} \hat{\zeta}^{j}\right] F_{t}^{k_{1} l_{1}} \ldots F_{t}^{k_{n-2} l_{n-2}} \lambda^{k_{n-1} l_{n-1}}\right\}= \\
& \quad-\epsilon_{i j k_{1} l_{1} \ldots k_{n-1} l_{n-1}} \hat{e}_{\infty}^{i}\left[\hat{D}_{\infty}\left(\hat{D}_{\infty} \hat{\zeta}^{j}\right)\right] F_{t}^{k_{1} l_{1}} \ldots F_{t}^{k_{n-2} l_{n-2}} \lambda^{k_{n-1} l_{n-1}}- \\
& \quad-(n-2) \epsilon_{i j k_{1} l_{1} \ldots k_{n-1} l_{n-1}} \hat{e}_{\infty}^{i}\left[\hat{D}_{\infty} \hat{\zeta}^{j}\right]\left[\hat{D}_{\infty} F_{t}^{k_{1} l_{1}}\right] F_{t}^{k_{2} l_{2}} \ldots F_{t}^{k_{n-2} l_{n-2}} \lambda^{k_{n-1} l_{n-1}}- \\
& \quad-\epsilon_{i j k_{1} l_{1} \ldots k_{n-1} l_{n-1}} \hat{e}_{\infty}^{i}\left[\hat{D}_{\infty} \hat{\zeta}^{j}\right] F_{t}^{k_{1} l_{1}} \ldots F_{t}^{k_{n-2} l_{n-2}}\left[\hat{D}_{\infty} \lambda^{k_{n-1} l_{n-1}}\right]
\end{aligned}
$$

and finally

$$
\begin{aligned}
& d\left[\epsilon_{i j k_{1} l_{1} \ldots k_{n-1} l_{n-1}} \hat{\zeta}_{\infty}^{i} \hat{\lambda}^{(-) j} F_{t}^{k_{1} l_{1}} \ldots F_{t}^{k_{n-1} l_{n-1}}\right]= \\
& =\epsilon_{i j k_{1} l_{1} \ldots k_{n-1} l_{n-1}}\left[\hat{D}_{\infty} \hat{\zeta}_{\infty}^{i}\right] \hat{\lambda}^{(-) j} F_{t}^{k_{1} l_{1}} \ldots F_{t}^{k_{n-1} l_{n-1}}- \\
& \quad-\epsilon_{i j k_{1} l_{1} \ldots k_{n-1} l_{n-1}} \hat{\zeta}_{\infty}^{i}\left[\hat{D}_{\infty} \hat{\lambda}^{(-) j}\right] F_{t}^{k_{1} l_{1}} \ldots F_{t}^{k_{n-1} l_{n-1}}- \\
& \quad-(n-1) \epsilon_{i j k_{1} l_{1} \ldots k_{n-1} l_{n-1}} \hat{\zeta}_{\infty}^{i} \hat{\lambda}^{(-) j}\left[\hat{D}_{\infty} F_{t}^{k_{1} l_{1}}\right] F_{t}^{k_{2} l_{2}} \ldots F_{t}^{k_{n-1} l_{n-1}}
\end{aligned}
$$

It is straightforward replace the previous expressions in eq. (6.3), discarding irrelevant total derivatives, to obtain an alternative expression of the AdS gauge anomaly that has no derivatives of the gauge parameter. An immediately apparent feature of that alternative expression of the anomaly is that, if the additional condition $\hat{D}_{\infty} \hat{\zeta}^{i}=0$ holds, then only the first term of the second member (the first line) is non zero. This condition, which for instance is automatically satisfied if the boundary manifold is of constant curvature, already appeared in ref. [38] as necessary for the finiteness of the action, which otherwise will have a divergence that would be linear on $r$ (or logarithmic in the Fefferman-Graham standard radial coordinate $\rho$ ).

\subsection{Weyl anomaly}

As said in the previous section, gauge transformations for which only $\lambda^{1}$ is non vanishing induce Weyl transformations at the boundary. The Weyl anomaly for the "Kounterterms" action principle that follows from eq. (6.3) is

$$
\Omega_{2 n}^{1}(A, \bar{A}, \lambda)=\kappa \epsilon_{k_{1} l_{1} \ldots k_{n} l_{n}} \hat{R}_{\infty}^{k_{1} l_{1}} \ldots \hat{R}_{\infty}^{k_{n} l_{n}} \lambda^{1}=\kappa E_{n} \lambda^{1}
$$

where $E_{n}=\epsilon_{k_{1} l_{1} \ldots k_{n} l_{n}} \hat{R}_{\infty}^{k_{1} l_{1}} \ldots \hat{R}_{\infty}^{k_{n} l_{n}}$ is the Euler density of the boundary (which is not required to vanish by the field equations). This result agrees with refs. [32-34].

\subsection{Lorentz and translational anomalies}

From eq. (6.3) we see that there is no Lorentz anomaly in the Kountereterms approach (unlike what happens in the Backgrounds approach).

The anomalies under gauge translations can be read from eq. (6.3), but as said above they vanish if the condition $\hat{D}_{\infty} \hat{\zeta}^{i}=0$ holds. This condition was found in ref. [38] to be necessary to ensure the finiteness of the action. 


\section{Diffeomorphism anomalies of Chern-Simons AdS gravity: backgrounds}

We will use eqs. (2.14) and (2.15) in this section. For the AdS vacuum $\left.I_{\xi}<\bar{F}^{n+1}\right\rangle=0$ and $\left\langle\bar{F}^{n} I_{\xi} \bar{A}>=0\right.$ as a result of the field equations. Also the asymptotic dependence of the relevant fields in the Backgrounds approach and the definition of the symmetric trace imply that $\left\langle\Delta A F_{t}^{n-1} I_{\xi} \bar{F}\right\rangle$. What remains has exactly the same form that the gauge anomaly for backgrounds, but with the "effective gauge parameter" $I_{\xi} \bar{A}$ instead of $\lambda$. From

$$
I_{\xi} \bar{A}=e^{r} I_{\xi} \hat{e}_{\infty}^{i}\left(P_{i}-J_{1 i}\right)+\frac{1}{2} I_{\xi} \hat{\omega}_{\infty}^{i j} J_{i j}+I_{\xi}(d r) P_{1}-\frac{1}{2} e^{-r} I_{\xi} \hat{\bar{\zeta}}^{i}\left(P_{i}+J_{1 i}\right),
$$

we can read the corresponding components of that "effective $\lambda$ " and just replace them in eq. (5.6) to obtain the diffeomorphism anomaly for Chern-Simons AdS gravity in the backgrounds approach. One important difference is that the components of this effective gauge parameter are not all independent, as once $\hat{e}_{\infty}^{i}$ is given then $\hat{\omega}_{\infty}^{i j}$ and then $\hat{\bar{\zeta}}^{i}$ are determined.

\section{Diffeomorphism anomalies of Chern-Simons AdS gravity: "Kountert- erms"}

We will use eqs. (2.14) and (2.15) as our starting point. The first observation is that for the "Kounterterms" vacuum $I_{\xi}<\bar{F}^{n+1}>=0$, as $\bar{F}$ only has components along $J_{i j}$. We have that $I_{\xi} \bar{F}=\frac{1}{2} I_{\xi} \hat{R}_{\infty}^{i j} J_{i j}$ and $I_{\xi} \bar{A}=\frac{1}{2} I_{\xi} \hat{\omega}_{\infty}^{i j} J_{i j}+I_{\xi}(d r) P_{1}$. Looking at each term of $\Omega_{2 n}^{1}(A, \bar{A}, \xi)$ we see that:

i. $\left\langle\bar{F}^{n} I_{\xi} \bar{A}>\right.$ is non zero if the $\bar{F}$ 's are along $J_{i j}$ and $I_{\xi} \bar{A}$ along $P_{1}$.

ii. $<\Delta A F_{t}^{n-1} I_{\xi} \bar{F}>$ has a non zero contribution if $\Delta A$ is along $\left(P_{i}-J_{1 i}\right)$, one $F_{t}$ is along $\left(P_{i}+J_{1 i}\right)$, the remaining $F_{t}$ 's along $J_{i j}$, and $I_{\xi} \bar{F}$ along $J_{i j}$.

iii. $<\Delta A F_{t}^{n-1} \bar{D} I_{\xi} \bar{A}>$ appears in a term that has exactly the same form that the last term in the gauge anomaly, with $I_{\xi} \bar{A}$ instead of $\lambda$. It follows that the discussion done for gauge anomalies apply. Notice that the "effective gauge parameter" $I_{\xi} \bar{A}$ does not contain gauge translations, and that the non vanishing components have the same asymptotic dependence that we required for $\lambda$.

Putting all together, the explicit form of the diffeomorphism anomaly is

$$
\begin{aligned}
& \Omega_{2 n}^{1}(A, \bar{A}, \xi)=\kappa \epsilon_{k_{1} l_{1} \ldots k_{n} l_{n}} \hat{R}_{\infty}^{k_{1} l_{1}} \ldots \hat{R}_{\infty}^{k_{n} l_{n}} I_{\xi}(d r)+ \\
& +2 n \kappa \int_{0}^{1} d t(t-1) t \epsilon_{i j k_{1} l_{1} \ldots k_{n-1} l_{n-1}} \hat{e}_{\infty}^{i}\left[\hat{D}_{\infty} \hat{\zeta}^{j}\right] F_{t}^{k_{1} l_{1}} \ldots F_{t}^{k_{n-2} l_{n-2}}\left[I_{\xi} \hat{R}_{\infty}^{k_{n-1} l_{n-1}}\right]+ \\
& +2 n \kappa \int_{0}^{1} d t(t-1) t \epsilon_{i j k_{1} l_{1} \ldots k_{n-1} l_{n-1}} \hat{e}_{\infty}^{i}\left[\hat{D}_{\infty} \hat{\zeta}^{j}\right] F_{t}^{k_{1} l_{1}} \ldots F_{t}^{k_{n-2} l_{n-2}} \hat{D}_{\infty}\left(I_{\xi} \hat{\omega}_{\infty}\right)^{k_{n-1} l_{n-1}} .
\end{aligned}
$$

Notice that, as it was the case for gauge anomalies, if the condition $\hat{D}_{\infty} \hat{\zeta}_{\infty}^{i}=0$ holds the only term of the second member that survives is the first. That term corresponds to the Weyl anomaly, as $I_{\xi}(d r)$ is non vanishing only for $\xi=\sigma \frac{\partial}{\partial r}$ where $\sigma$ is an infinitesimal parameter (or function) for infinitesimal coordinate transformations, and it is well 
known [47], and easy to check, that radial diffeomorphisms induce Weyl transformations at the boundary. In that case $I_{\xi}(d r)=\sigma$ and the Weyl anomaly is

$$
\Omega_{2 n}^{1}(A, \bar{A}, \xi)=\kappa \epsilon_{k_{1} l_{1} \ldots k_{n} l_{n}} \hat{R}_{\infty}^{k_{1} l_{1}} \ldots \hat{R}_{\infty}^{k_{n} l_{n}} \sigma,
$$

again in agreement with refs. [32-34].

\section{Discussion and comments}

We have computed AdS gauge and diffeomorphism anomalies for Chern-Simons AdS gravity, for two action principles discussed in previous works [18, 19, 38], and assuming the asymptotic behavior of the fundamental fields proposed in [38]. The anomalies arise as a result of considering the second field configuration, taken as a regulator, as non varying. This means in particular that if one regards both $A$ and $\bar{A}$ as dynamical fields varying with the same transformation rules there would be no anomalies at all.

The results are finite without requiring further regularization or subtraction, adding to the evidence that the action principles motivated by the transgression are indeed the appropriate ones.

Anomalies are characterized by a symmetric tensor (or equivalently a symmetric trace) in the algebra of the relevant gauge group and an overall constant factor (see for instance $[20,23])$. The anomalies found here, in particular Lorentz or gravitational anomalies, involve the Levi-Civita tensor as the invariant trace. On the other hand, standard gravitational anomalies for theories with chiral fermions or (anti)self dual antisymmetric tensors with the standard minimal coupling to gravity are given in terms of standard traces properly symmetrized [21, 23]. This is somewhat puzzling, as it is not clear what sort of hypothetical dual CFT theory would yield matching anomalies.

Gravitational anomalies of the standard form have been studied in the context of AdSCFT correspondence, for instance in [48, 49]. However these anomalies are generated by adding Lorentz Chern-Simons terms in the bulk, constructed with a symmetric standard trace instead of the Levi-Civita tensor. It seems that to generate gravitational anomalies of the standard form one should start with a Lagrangian of the kind known as "exotic Chern-Simons gravity", where the invariant tensor in the AdS gauge group algebra is a symmetrized combination of standard traces, instead of the Levi-Civita tensor. It would be interesting to do an analysis similar to [38] for "exotic CS gravity" and then compute the AdS anomalies that could arise in that case.

Another interesting set of questions has to do with the role of torsion in the anomalies computed. The analysis of [38] showed that the intrinsic torsion of the boundary must vanish if the AdS gauge curvature is asymptotically finite. However the bulk torsion itself is not required to vanish, and for instance $\zeta^{i}$ contains information about it. It would be valuable to understand better the physical implications of the bulk torsion contribution to the anomalies computed. It would be interesting to see if the anomalies discussed here are related to the ones discussed in [50], for a different kind of theory and in $2+1$ dimensions.

Anomalies involving space-time torsion of the kind introduced in [51, 52] are excluded in our framework, as we have a vanishing boundary torsion as an asymptotic condition. 
Furthermore those anomalies involve standard traces, while the symmetric trace we are considering involves contractions with the Levi-Civita tensor. However they may appear in a holographic context involving "exotic CS gravity", that may admit a different asymptotic torsion.

The possible forms of anomalies are quite constrained by the Wess-Zumino (WZ) consistency conditions (see for instance [20,21]), which means that anomalies of the form discussed here may appear may be relevant for other field theories. In particular, they may be relevant for Lovelock gravity theories and their holographic dual theories. ${ }^{16}$ It has been proved that the boundary term suggested by transgressions [18, 19], with a suitable constant coefficient, also works as a regulator for General Relativity with a cosmological constant in odd dimensions [45], and in fact for any Lovelock AdS gravity [42, 57]. That is surprising at first, but maybe the fact that the variation of the boundary term (which in fact has information about the bulk) would generate anomalies satisfying the Wess-Zumino consistency condition may explain that fact.

\section{Acknowledgments}

I am grateful to M. Reisenberger for reading the manuscript and making many valuable suggestions. I am grateful to O. Miskovic and R. Olea for enlightening discussions and comments. I acknowledge financial support from the Sistema Nacional de Investigadores (SNI), Uruguay, while part of this work was done.

Open Access. This article is distributed under the terms of the Creative Commons Attribution License (CC-BY 4.0), which permits any use, distribution and reproduction in any medium, provided the original author(s) and source are credited.

\section{References}

[1] A. Achucarro and P.K. Townsend, A Chern-Simons action for three-dimensional anti-de Sitter supergravity theories, Phys. Lett. B 180 (1986) 89 [INSPIRE].

[2] E. Witten, (2+1)-dimensional gravity as an exactly soluble system, Nucl. Phys. B 311 (1988) 46 [INSPIRE].

[3] A.H. Chamseddine, Topological gauge theory of gravity in five-dimensions and all odd dimensions, Phys. Lett. B 233 (1989) 291 [INSPIRE].

[4] A.H. Chamseddine, Topological gravity and supergravity in various dimensions, Nucl. Phys. B 346 (1990) 213 [INSPIRE].

[5] M. Bañados, R. Troncoso and J. Zanelli, Higher dimensional Chern-Simons supergravity, Phys. Rev. D 54 (1996) 2605 [gr-qc/9601003] [InSPIRE].

[6] R. Troncoso and J. Zanelli, New gauge supergravity in seven-dimensions and eleven-dimensions, Phys. Rev. D 58 (1998) 101703 [hep-th/9710180] [INSPIRE].

\footnotetext{
${ }^{16}$ Lovelock gravity theories $[53,54]$ are a class of gravity theories that were shown $[55,56]$ to be possible low energy effective descriptions of gravity in string theory. These theories have been extensively studied afterwards.
} 
[7] J. Zanelli, Lecture notes on Chern-Simons (super-)gravities, hep-th/0502193 [INSPIRE].

[8] R. Aros, M. Contreras,R. Olea, R. Troncoso and J. Zanelli, Charges in $2+1$ Dimensional Gravity and Supergravity, presented at the Strings'99 Conference, Potsdam Germany (1999).

[9] P. Mora and H. Nishino, Fundamental extended objects for Chern-Simons supergravity, Phys. Lett. B 482 (2000) 222 [hep-th/0002077] [INSPIRE].

[10] P. Mora, Chern-Simons supersymmetric branes, Nucl. Phys. B 594 (2001) 229 [hep-th/0008180] [INSPIRE].

[11] A. Borowiec, M. Ferraris and M. Francaviglia, A covariant formalism for Chern-Simons gravity, J. Phys. A 36 (2003) 2589 [hep-th/0301146] [INSPIRE].

[12] A. Borowiec, L. Fatibene, M. Ferraris and M. Francaviglia, Covariant Lagrangian formulation of Chern-Simons and BF theories, Int. J. Geom. Meth. Mod. Phys. 3 (2006) 755 [hep-th/0511060] [INSPIRE].

[13] F. Izaurieta, E. Rodriguez and P. Salgado, On transgression forms and Chern-Simons (super)gravity, hep-th/0512014 [INSPIRE].

[14] F. Izaurieta, E. Rodriguez and P. Salgado, The extended Cartan homotopy formula and a subspace separation method for Chern-Simons supergravity, Lett. Math. Phys. 80 (2007) 127 [hep-th/0603061] [INSPIRE].

[15] G. Sardanashvily, Gauge conservation laws in higher dimensional Chern-Simons models, hep-th/0303059 [INSPIRE].

[16] G. Sardanashvily, Energy momentum conservation laws in higher dimensional Chern-Simons models, hep-th/0303148 [INSPIRE].

[17] P. Mora, Transgressión forms as unifying principle in field theory, hep-th/0512255 [INSPIRE].

[18] P. Mora, R. Olea, R. Troncoso and J. Zanelli, Finite action principle for Chern-Simons AdS gravity, JHEP 06 (2004) 036 [hep-th/0405267] [INSPIRE].

[19] P. Mora, R. Olea, R. Troncoso and J. Zanelli, Transgression forms and extensions of Chern-Simons gauge theories, JHEP 02 (2006) 067 [hep-th/0601081] [InSPIRE].

[20] B. Zumino, Y.-S. Wu and A. Zee, Chiral anomalies, higher dimensions and differential geometry, Nucl. Phys. B 239 (1984) 477 [inSPIRE].

[21] W.A. Bardeen and B. Zumino, Consistent and covariant anomalies in gauge and gravitational theories, Nucl. Phys. B 244 (1984) 421 [INSPIRE].

[22] J. Manes, R. Stora and B. Zumino, Algebraic study of chiral anomalies, Commun. Math. Phys. 102 (1985) 157 [INSPIRE].

[23] L. Alvarez-Gaumé and P. Ginsparg, The structure of gauge and gravitational anomalies, Annals Phys. 161 (1985) 423.

[24] J.M. Maldacena, The large- $N$ limit of superconformal field theories and supergravity, Int. J. Theor. Phys. 38 (1999) 1113 [Adv. Theor. Math. Phys. 2 (1998) 231] [hep-th/9711200] [INSPIRE].

[25] E. Witten, Anti-de Sitter space and holography, Adv. Theor. Math. Phys. 2 (1998) 253 [hep-th/9802150] [INSPIRE]. 
[26] S.S. Gubser, I.R. Klebanov and A.M. Polyakov, Gauge theory correlators from noncritical string theory, Phys. Lett. B 428 (1998) 105 [hep-th/9802109] [InSPIRE].

[27] M. Henningson and K. Skenderis, The holographic Weyl anomaly, JHEP 07 (1998) 023 [hep-th/9806087] [INSPIRE].

[28] M.J. Duff, Twenty years of the Weyl anomaly, Class. Quant. Grav. 11 (1994) 1387 [hep-th/9308075] [INSPIRE].

[29] S. Nojiri and S.D. Odintsov, On the conformal anomaly from higher derivative gravity in AdS/CFT correspondence, Int. J. Mod. Phys. A 15 (2000) 413 [hep-th/9903033] [InSPIRE].

[30] M. Blau, K.S. Narain and E. Gava, On subleading contributions to the AdS/CFT trace anomaly, JHEP 09 (1999) 018 [hep-th/9904179] [INSPIRE].

[31] A. Schwimmer and S. Theisen, Universal features of holographic anomalies, JHEP 10 (2003) 001 [hep-th/0309064] [INSPIRE].

[32] M. Bañados, A. Schwimmer and S. Theisen, Chern-Simons gravity and holographic anomalies, JHEP 05 (2004) 039 [hep-th/0404245] [INSPIRE].

[33] M. Bañados, O. Mišković and S. Theisen, Holographic currents in first order gravity and finite Fefferman-Graham expansions, JHEP 06 (2006) 025 [hep-th/0604148] [INSPIRE].

[34] M. Bañados, R. Olea and S. Theisen, Counterterms and dual holographic anomalies in CS gravity, JHEP 10 (2005) 067 [hep-th/0509179] [INSPIRE].

[35] A. Schwimmer and S. Theisen, Entanglement Entropy, Trace Anomalies and Holography, Nucl. Phys. B 801 (2008) 1 [arXiv:0802.1017] [INSPIRE].

[36] N. Boulanger, General solutions of the Wess-Zumino consistency condition for the Weyl anomalies, JHEP 07 (2007) 069 [arXiv:0704.2472] [INSPIRE].

[37] N. Boulanger, Algebraic Classification of Weyl Anomalies in Arbitrary Dimensions, Phys. Rev. Lett. 98 (2007) 261302 [arXiv:0706.0340] [INSPIRE].

[38] P. Mora, Action Principles for Transgression and Chern-Simons AdS Gravities, JHEP 11 (2014) 128 [arXiv:1407.6032] [INSPIRE].

[39] C. Fefferman and R. Graham, Conformal Invariants, in The mathematical heritage of Elie Cartan Conference, Lyon France (1984), Astérisque Numero Hors Serie (1985) 95.

[40] P. Mora, Transgressions and Holographic Conformal Anomalies for Chern-Simons Gravities, arXiv: 1010.5110 [INSPIRE].

[41] A. Das, B. Mukhopadhyaya and S. SenGupta, Why has spacetime torsion such negligible effect on the Universe?, Phys. Rev. D 90 (2014) 107901 [arXiv:1410.0814] [INSPIRE].

[42] R. Olea, Regularization of odd-dimensional AdS gravity: Kounterterms, JHEP 04 (2007) 073 [hep-th/0610230] [INSPIRE].

[43] I.G. Moss, Anomalies, boundaries and the in-in formalism, J. Phys. A 45 (2012) 374022 [arXiv: 1201.5732] [INSPIRE].

[44] M. Nakahara, Geometry, Topology and Physics, IOP, Bristol U.K. (1991).

[45] P. Mora, R. Olea, R. Troncoso and J. Zanelli, Vacuum energy in odd-dimensional AdS gravity, hep-th/0412046 [INSPIRE].

[46] H.R. Afshar, Flat/AdS boundary conditions in three dimensional conformal gravity, JHEP 10 (2013) 027 [arXiv: 1307.4855] [inSPIRE]. 
[47] C. Imbimbo, A. Schwimmer, S. Theisen and S. Yankielowicz, Diffeomorphisms and holographic anomalies, Class. Quant. Grav. 17 (2000) 1129 [hep-th/9910267] [INSPIRE].

[48] P. Kraus and F. Larsen, Holographic gravitational anomalies, JHEP 01 (2006) 022 [hep-th/0508218] [INSPIRE].

[49] S.N. Solodukhin, Holographic description of gravitational anomalies, JHEP 07 (2006) 003 [hep-th/0512216] [INSPIRE].

[50] M. Blagojevic, B. Cvetkovic, O. Mišković and R. Olea, Holography in 3D AdS gravity with torsion, JHEP 05 (2013) 103 [arXiv:1301.1237] [INSPIRE].

[51] O. Chandía and J. Zanelli, Topological invariants, instantons and chiral anomaly on spaces with torsion, Phys. Rev. D 55 (1997) 7580 [hep-th/9702025] [INSPIRE].

[52] O. Chandía and J. Zanelli, Supersymmetric particle in a space-time with torsion and the index theorem, Phys. Rev. D 58 (1998) 045014 [hep-th/9803034] [InSPIRE].

[53] D. Lovelock, The Einstein tensor and its generalizations, J. Math. Phys. 12 (1971) 498 [INSPIRE].

[54] D. Lovelock, The four-dimensionality of space and the Einstein tensor, J. Math. Phys. 13 (1972) 874 [INSPIRE].

[55] B. Zwiebach, Curvature Squared Terms and String Theories, Phys. Lett. B 156 (1985) 315 [INSPIRE].

[56] B. Zumino, Gravity theories in more than four-dimensions, Phys. Rept. 137 (1986) 109 [INSPIRE].

[57] G. Kofinas and R. Olea, Universal regularization prescription for Lovelock AdS gravity, JHEP 11 (2007) 069 [arXiv: 0708.0782] [INSPIRE]. 